\title{
Finite-Horizon Robust Kalman Filter for Uncertain Attitude Estimation System with Star Sensor Measurement Delays
}

\author{
Hua-Ming Qian, ${ }^{1}$ Wei Huang, ${ }^{1}$ and Biao Liu ${ }^{2}$ \\ ${ }^{1}$ College of Automation, Harbin Engineering University, Harbin 150001, China \\ ${ }^{2}$ Dongguan Techtotop Microelectronics Co., Ltd., Chengdu R \& D Branch, Chengdu 610041, China \\ Correspondence should be addressed to Wei Huang; huangwei2393@163.com
}

Received 10 October 2013; Accepted 6 January 2014; Published 23 February 2014

Academic Editor: Xiaojie Su

Copyright (c) 2014 Hua-Ming Qian et al. This is an open access article distributed under the Creative Commons Attribution License, which permits unrestricted use, distribution, and reproduction in any medium, provided the original work is properly cited.

\begin{abstract}
This paper addresses the robust Kalman filtering problem for uncertain attitude estimation system with star sensor measurement delays. Combined with the misalignment errors and scale factor errors of gyros in the process model and the misalignment errors of star sensors in the measurement model, the uncertain attitude estimation model can be established, which indicates that uncertainties not only appear in the state and output matrices but also affect the statistic of the process noise. Meanwhile, the phenomenon of star sensor measurement delays is described by introducing Bernoulli random variables with different delay characteristics. The aim of the addressed attitude estimation problem is to design a filter such that, in the presence of model uncertainties and star sensors delays for the attitude estimation system, the optimized filter parameters can be obtained to minimize the upper bound on the estimation error covariance. Therefore, a finite-horizon robust Kalman filter is proposed to cope with this question. Compared with traditional attitude estimation algorithms, the designed robust filter takes into account the effects of star sensor measurement delays and model uncertainties. Simulation results illustrate the effectiveness of the developed robust filter.
\end{abstract}

\section{Introduction}

Attitude estimation has played an important role in many actual applications, such as aerospace, satellites, marine, and robots. For attitude estimation system, due to the high measurement precision of star sensor, the rate gyro and star sensor are often integrated to determinate the spacecraft attitude. Furthermore, the filter design is one of the key technologies in attitude estimation. As is well known, Kalman filter has been employed to solve the attitude estimation filtering problem [1-3]. Although these attitude estimation filtering algorithms are available for handling attitude estimation problem, they need to know the accurate model with Gaussian noises and assume exact alignment of gyro and star sensor. However, in practical problems, the measurement misalignment errors of these sensors are inevitable, which will severely degrade the filtering performance. To overcome the sensor misalignment problem in attitude estimation, many researches have been reported in some recent notes [4-8]. For example, Shuster et al. [4] utilize the batch estimation technique to calibrate the misalignment of the sensors. Pittelkau $[5,6]$ develops the Kalman filtering technique to estimate the calibration parameters of gyro and star sensor. Lai and Crassidis [7] derived a new spacecraft sensor alignment estimation approach based on the unscented filter. Vandersteen [8] presents the real-time moving horizon estimation of a spacecraft's attitude and sensor calibration parameters. Unfortunately, even though misalignment calibration is accomplished, the measurement misalignment error of gyro and star sensor cannot be removed completely, which lead to model uncertainty. Therefore, in the case that an exact uncertain model is established, the robust filtering technique can be used to deal with the filtering problem with model uncertainty. For the purpose, in the past few decades, many researchers' attentions have been drawn to the robust filtering problem with model uncertainties, including the $H_{\infty}$ filter [9], new energy-to-peak FIR filter [10], fuzzy filter design [11], and robust Kalman filter [12,13]. Among them, the robust Kalman filter design based on the minimum variance theory has been approved to be an effective methodology. Based on 
this, Wang et al. [14] proposed a regularized robust filter for attitude determination system to deal with the installation error of star trackers. In this work, the installation error of star trackers is expressed as model uncertainty in the measurement model, but the measurement misalignment error of gyros is not taken into account.

In this paper, all the misalignment errors and scale factor errors of gyros introduced into the process model and the misalignment errors of star sensors in the measurement model are described as model uncertainties, so that uncertain attitude estimation model is established. From the uncertain model, we can find that the attitude estimation filtering problem suffers from uncertainties in the state and output matrices and uncertainty in the process noise matrix. A typical way is to represent the model uncertainties as normbounded uncertainties. Recently, the finite-horizon robust Kalman filter design has been investigated to be available for handling the filtering problem with model uncertainties in the state, output, and noise matrices by getting an optimized upper bound on the estimation error covariance [15]. Souto and Ishihara [16] extend this work by considering correlated noises with unknown mean and variance.

However, the above works have been based on this assumption that all the observations should be available at the time of estimation. In many situations, the sensor measurements are disturbed by complicated signal processing circuit, leading to the sensor measurement delays or measurement failures [17-20]. Therefore, the filtering problems with sensor measurement delays have stirred considerable research attention, such as [21-26]. In attitude estimation system, due to optics imaging, star recognition, and attitude determination, the attitude information output of star sensors has the random delay characteristic. Up to now, the attitude estimation filtering problem with model uncertainties in the state, output, and process noise matrices and star sensor delays has not been reported. So, there is great desire to present a robust Kalman filter for uncertain attitude estimation system with star sensor delays.

Based on the above discussion, a finite-horizon robust Kalman filter is proposed for uncertain attitude estimation system with star sensor delays. The star sensor measurement is assumed as one-step randomly delayed measurement with different delay characteristics. The main contributions of the paper are as follows. (1) The uncertain attitude estimation model is established to take into consideration measurement errors of gyros and star sensors, which indicates that the norm-bounded uncertainties appear in the state, output, and process noise matrices. (2) Combined with star sensor delays, a new finite-horizon robust Kalman filter design is derived for the uncertain attitude estimation system. (3) The Hadamard product is employed to help the robust Kalman filter development. (4) The presented robust filter is recursive, which is suitable for online applications.

This paper is organized as follows. In Section 2, the uncertainty attitude estimation model with star sensor delays is set up. In Section 3, a finite-horizon robust Kalman filter for uncertainty attitude estimation system with star sensor delays is developed. In Section 4, the simulation results and analysis are given. In Section 5, some conclusions are drawn.

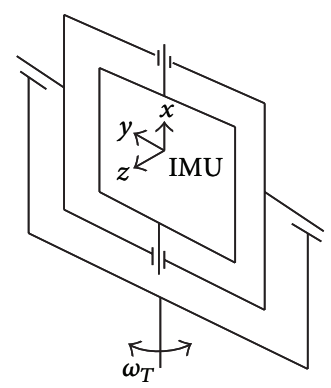

FIgURE 1: A brief principal diagram of the gyro.

\section{Uncertainty Attitude Estimation Model with Star Sensor Delays}

2.1. Gyro Error Model. As Figure 1 shows, $x, y$, and $z$ are three axes of the gyro, respectively. The gyro model with misalignment errors and scale factor errors is given as follows:

$$
\begin{gathered}
\widetilde{\boldsymbol{\omega}}=\left(\mathbf{I}_{3 \times 3}+\mathbf{M}\right) \boldsymbol{\omega}+\boldsymbol{\beta}+\boldsymbol{\eta}_{v} \\
\dot{\boldsymbol{\beta}}=\boldsymbol{\eta}_{u},
\end{gathered}
$$

where $\widetilde{\boldsymbol{\omega}}$ is the gyro measured output, $\boldsymbol{\omega}$ is the actual gyro angular rate, $\boldsymbol{\beta}$ is the gyro bias, $\boldsymbol{\eta}_{v}$ and $\boldsymbol{\eta}_{u}$ are independent Gaussian white-noise processes with zero means and covariance $\sigma_{v}^{2}$ and covariance $\sigma_{u}^{2}$, and $\mathbf{M}$ is an unknown matrix with misalignment errors and scale factor errors, which is defined by

$$
\mathbf{M}=\left[\begin{array}{lll}
\lambda_{x} & \delta_{x y} & \delta_{x z} \\
\delta_{y x} & \lambda_{y} & \delta_{y z} \\
\delta_{z x} & \delta_{z y} & \lambda_{z}
\end{array}\right],
$$

where $\lambda=\left[\begin{array}{lll}\lambda_{x} & \lambda_{y} & \lambda_{z}\end{array}\right]^{T}$ is the unknown scale factor error vector and $\delta_{i j}$ is the projection of the $i$-gyro axis on the $j$ body-axis, which is assumed to be a small and unknown misalignment angle.

2.2. Uncertainty Process Model. The quaternion is employed to express the attitude for the attitude estimation system consisting of the gyro and star tracker. So, the quaternion orientation equation is described as

$$
\dot{\mathbf{q}}=\frac{1}{2}\left[\begin{array}{c}
\boldsymbol{\omega} \\
0
\end{array}\right] \otimes \mathbf{q}=\frac{1}{2} \boldsymbol{\Omega}(\boldsymbol{\omega}) \cdot \mathbf{q},
$$

where $\mathbf{q}=\left[\begin{array}{llll}q_{1} & q_{2} & q_{3} & q_{4}\end{array}\right]^{T}=\left[\begin{array}{ll}\boldsymbol{\rho}^{T} & q_{4}\end{array}\right]^{T}$ is the attitude quaternion, $\boldsymbol{\rho}$ is the quaternion vector, $q_{4}$ is the quaternion scalar part, $\otimes$ is the quaternion product, and $\boldsymbol{\Omega}(\boldsymbol{\omega})$ can be defined as follows:

$$
\boldsymbol{\Omega}(\boldsymbol{\omega})=\left[\begin{array}{cc}
-[\boldsymbol{\omega} \times] & \boldsymbol{\omega} \\
-\boldsymbol{\omega}^{T} & 0
\end{array}\right],
$$

where $[\boldsymbol{\omega} \times]$ is a cross-product matrix defined by

$$
[\boldsymbol{\omega} \times]=\left[\begin{array}{ccc}
0 & -\omega_{3} & \omega_{2} \\
\omega_{3} & 0 & -\omega_{1} \\
-\omega_{2} & \omega_{1} & 0
\end{array}\right] .
$$


Using quaternion multiplication, the quaternion error is expressed as

$$
\delta \mathbf{q}=\mathbf{q} \otimes \widehat{\mathbf{q}}^{-1}=\left[\begin{array}{ll}
\Delta \boldsymbol{\rho}^{T} & \Delta q_{4}
\end{array}\right]^{T},
$$

where $\mathbf{q}$ is the true quaternion, $\widehat{\mathbf{q}}$ is the estimated quaternion, $\mathbf{q}^{-1}$ is the inverse quaternion, which is given by $\mathbf{q}^{-1}=$ $\left[\begin{array}{ll}-\boldsymbol{\rho}^{T} & q_{4}\end{array}\right]^{T}$, and $\Delta \boldsymbol{\rho}$ is the quaternion error vector part.

The gyro error angular rate $\delta \omega$ is assumed as the difference between the estimated and actual angular rate: $\delta \boldsymbol{\omega}=$ $\boldsymbol{\omega}-\widehat{\boldsymbol{\omega}}$. According to (1), using a small angle approximation, we have

$$
\begin{aligned}
\delta \boldsymbol{\omega} & =\boldsymbol{\omega}-\widehat{\boldsymbol{\omega}}=\boldsymbol{\omega}-\left(\mathbf{I}_{3 \times 3}+\mathbf{M}\right)^{-1}(\widetilde{\boldsymbol{\omega}}-\widehat{\boldsymbol{\beta}}) \\
& =\boldsymbol{\omega}-\left(\mathbf{I}_{3 \times 3}+\mathbf{M}\right)^{-1}\left[\left(\mathbf{I}_{3 \times 3}+\mathbf{M}\right) \boldsymbol{\omega}+\boldsymbol{\beta}+\boldsymbol{\eta}_{v}-\widehat{\boldsymbol{\beta}}\right] \\
& =-\left(\mathbf{I}_{3 \times 3}+\mathbf{M}\right)^{-1}\left(\Delta \boldsymbol{\beta}+\boldsymbol{\eta}_{v}\right) \approx-\left(\mathbf{I}_{3 \times 3}-\mathbf{M}\right)\left(\Delta \boldsymbol{\beta}+\boldsymbol{\eta}_{v}\right),
\end{aligned}
$$

where $\Delta \boldsymbol{\beta}$ is the gyro bias error vector.

Differentiating (6) with respect to time and combining the quaternion multiplication, we obtain

$$
\begin{aligned}
\delta \dot{\mathbf{q}} & =\dot{\mathbf{q}} \otimes \widehat{\mathbf{q}}^{-1}+\mathbf{q} \otimes \dot{\overrightarrow{\mathbf{q}}}^{-1}=\dot{\mathbf{q}} \otimes \widehat{\mathbf{q}}^{-1}+\mathbf{q} \otimes \frac{1}{2} \widehat{\mathbf{q}}^{-1} \otimes\left[\begin{array}{c}
\widehat{\boldsymbol{\omega}} \\
0
\end{array}\right]^{-1} \\
& =\frac{1}{2}\left[\begin{array}{c}
\boldsymbol{\omega} \\
0
\end{array}\right] \otimes \delta \mathbf{q}-\frac{1}{2} \delta \mathbf{q} \otimes\left[\begin{array}{c}
\widehat{\boldsymbol{\omega}} \\
0
\end{array}\right] \\
& =\frac{1}{2}\left[\begin{array}{c}
\widehat{\boldsymbol{\omega}} \\
0
\end{array}\right] \otimes \delta \mathbf{q}-\frac{1}{2} \delta \mathbf{q} \otimes\left[\begin{array}{c}
\widehat{\boldsymbol{\omega}} \\
0
\end{array}\right]+\frac{1}{2}\left[\begin{array}{c}
\delta \boldsymbol{\omega} \\
0
\end{array}\right] \otimes \delta \mathbf{q} \\
& =\left[\begin{array}{c}
-[\widehat{\boldsymbol{\omega}} \times] \Delta \boldsymbol{\rho} \\
0
\end{array}\right]+\frac{1}{2}\left[\begin{array}{c}
\Delta q_{4} \cdot \delta \boldsymbol{\omega}-[\delta \boldsymbol{\omega} \times] \Delta \boldsymbol{\rho} \\
-\delta \boldsymbol{\omega}^{T} \Delta \boldsymbol{\rho}
\end{array}\right] .
\end{aligned}
$$

In order to avoid the quaternion normalization constraint, only the vector component of the quaternion error $\delta \mathbf{q}$ is considered in the states. Inserting (7) into (8), by neglecting the second-order terms, we have

$$
\begin{aligned}
\Delta \dot{\boldsymbol{\rho}} & =-[\widehat{\boldsymbol{\omega}} \times] \Delta \boldsymbol{\rho}+\frac{1}{2} \delta \boldsymbol{\omega} \\
& =-[\widehat{\boldsymbol{\omega}} \times] \Delta \boldsymbol{\rho}-\frac{1}{2}\left(\mathbf{I}_{3 \times 3}-\mathbf{M}\right)\left(\Delta \boldsymbol{\beta}+\boldsymbol{\eta}_{v}\right) .
\end{aligned}
$$

The quaternion error vector part $\Delta \rho$ and the gyro bias error vector $\Delta \boldsymbol{\beta}$ are constructed as the error state vector: $\mathbf{x}=$ $\left[\begin{array}{ll}\Delta \boldsymbol{\rho}^{T} & \Delta \boldsymbol{\beta}^{T}\end{array}\right]^{T}$. An error state process model with unknown misalignment errors and scale factor errors can be expressed as

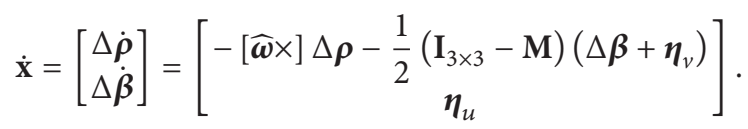

According to (10), the discrete-time process equation can be developed as

$$
\mathbf{x}_{k+1}=\left(\mathbf{A}_{k}+\Delta \mathbf{A}_{k}\right) \mathbf{x}_{k}+\left(\mathbf{B}_{k}+\Delta \mathbf{B}_{k}\right) \mathbf{w}_{k},
$$

where $\mathbf{w}_{k}$ is the zero mean Gaussian noise with covariance $\mathbf{Q}_{k}=\left[\begin{array}{cc}\Delta t \sigma_{v}^{2} \mathbf{I}_{3 \times 3} & \mathbf{0}_{3 \times 3} \\ \mathbf{0}_{3 \times 3} & \Delta t \sigma_{u}^{2} \mathbf{I}_{3 \times 3}\end{array}\right]$,

$$
\begin{array}{cc}
\mathbf{A}_{k}=\left[\begin{array}{cc}
\mathbf{I}_{3 \times 3}-[\widehat{\boldsymbol{\omega}} \times] & -\frac{1}{2} \mathbf{I}_{3 \times 3} \\
\mathbf{0}_{3 \times 3} & \mathbf{I}_{3 \times 3}
\end{array}\right], \quad \Delta \mathbf{A}_{k}=\left[\begin{array}{cc}
\mathbf{0}_{3 \times 3} & \frac{1}{2} \mathbf{M} \\
\mathbf{0}_{3 \times 3} & \mathbf{0}_{3 \times 3}
\end{array}\right], \\
\mathbf{B}_{k}=\left[\begin{array}{cc}
-\frac{1}{-} \mathbf{I}_{3 \times 3} & \mathbf{0}_{3 \times 3} \\
\mathbf{0}_{3 \times 3} & \mathbf{I}_{3 \times 3}
\end{array}\right], & \Delta \mathbf{B}_{k}=\left[\begin{array}{cc}
\frac{1}{2} \mathbf{M} & \mathbf{0}_{3 \times 3} \\
\mathbf{0}_{3 \times 3} & \mathbf{0}_{3 \times 3}
\end{array}\right] .
\end{array}
$$

From (11), it can be seen that the unknown error matrix $\mathbf{M}$ not only appears in the state matrix but also affects the statistic of the process noise. In order to realize the robust filtering design, the unknown error matrix $\mathbf{M}$ can be rewritten as

$$
M=\text { HFE, }
$$

where $\delta_{i j}=\Delta_{i j} \sigma_{i j}(i, j=x, y, z$, and $i \neq j) ; \lambda_{i}=\gamma_{i} \Delta \lambda_{i}(i=$ $x, y, z)$,

$$
\begin{aligned}
& \mathbf{H}=\left[\begin{array}{llllllll}
\gamma_{x} & \sigma_{x y} & \sigma_{x z} & & & & & \\
& & & \sigma_{y x} & \gamma_{y} & \sigma_{y z} & & \\
& & & & & \sigma_{z x} & \sigma_{z y} & \gamma_{z}
\end{array}\right] \\
& \mathbf{F}=\operatorname{diag}\left(\left[\begin{array}{lllllllll}
\Delta \lambda_{1} & \Delta_{x y} & \Delta_{x z} & \Delta_{y x} & \Delta \lambda_{2} & \Delta_{y z} & \Delta_{z x} & \Delta_{z y} & \Delta \lambda_{3}
\end{array}\right]\right) \\
& \mathbf{E}=\left[\begin{array}{lllllllll}
1 & 0 & 0 & 1 & 0 & 0 & 1 & 0 & 0 \\
0 & 1 & 0 & 0 & 1 & 0 & 0 & 1 & 0 \\
0 & 0 & 1 & 0 & 0 & 1 & 0 & 0 & 1
\end{array}\right]^{T}
\end{aligned}
$$

The parameters $\sigma_{i j}$ and $\gamma_{i}$ are positive constants, which can be chosen by the priori information of the gyro installation errors. If the $\sigma_{i j}$ and $\gamma_{i}$ are set to be large enough, the inequalities $\Delta_{i j} \Delta_{i j}^{T} \leq 1$ and $\Delta \lambda_{i} \Delta \lambda_{i}^{T} \leq 1$ can be fulfilled, so that the inequality $\mathbf{F F}^{T} \leq \mathbf{I}$ is satisfied. According to (13), the model error matrices $\Delta \mathbf{A}_{k}$ and $\Delta \mathbf{B}_{k}$ can be described as

$$
\Delta \mathbf{A}_{k}=\mathbf{H}_{1, k} \mathbf{F}_{1, k} \mathbf{E}_{1, k}, \quad \Delta \mathbf{B}_{k}=\mathbf{H}_{1, k} \mathbf{F}_{1, k} \mathbf{E}_{2, k},
$$

where $\mathbf{F}_{1, k} \mathbf{F}_{1, k}^{T} \leq \mathbf{I}$,

$$
\begin{array}{cc}
\mathbf{H}_{1, k}=\left[\begin{array}{cc}
\frac{1}{2} \mathbf{H} & \\
& \mathbf{0}_{3 \times 9}
\end{array}\right], & \mathbf{F}_{1, k}=\left[\begin{array}{ll}
\mathbf{F} & \\
& \mathbf{0}_{9 \times 9}
\end{array}\right], \\
\mathbf{E}_{1, k}=\left[\begin{array}{cc}
\mathbf{0}_{9 \times 3} & \mathbf{E} \\
\mathbf{0}_{9 \times 3} & \mathbf{0}_{9 \times 3}
\end{array}\right], & \mathbf{E}_{2, k}=\left[\begin{array}{cc}
\mathbf{E} & \mathbf{0}_{9 \times 3} \\
\mathbf{0}_{9 \times 3} & \mathbf{0}_{9 \times 3}
\end{array}\right] .
\end{array}
$$

2.3. Uncertainty Measurement Model with Star Sensor Delays. To obtain the attitude information, three star sensors are chosen. Considering the misalignment error of star sensors, the measurement model with model errors is expressed as

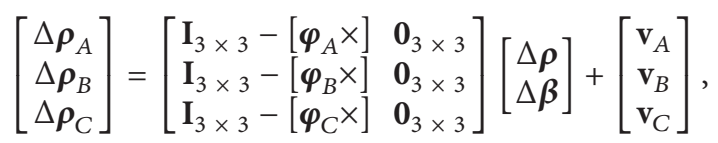


where $A, B$, and $C$ denote different star sensors, $\Delta \boldsymbol{\rho}_{i}(i=$ $A, B, C)$ are the measured quaternion error vector parts of star sensors, $\varphi_{i}=\left[\begin{array}{lll}\varphi_{i x} & \varphi_{i y} & \varphi_{i z}\end{array}\right]^{T}(i=A, B, C)$ is the unknown misalignment error vector, and $\mathbf{v}_{i}(i=A, B, C)$ are the zero mean Gaussian white noises with covariance matrix $\sigma_{s}^{2} \mathbf{I}_{3 \times 3}$. The unknown cross-product matrix $\left[\varphi_{i} \times\right](i=A, B, C)$ can be written as

$$
-\left[\boldsymbol{\varphi}_{i} \times\right]=-\left[\begin{array}{ccc}
0 & -\varphi_{i z} & \varphi_{i y} \\
\varphi_{i z} & 0 & -\varphi_{i x} \\
-\varphi_{i y} & \varphi_{i x} & 0
\end{array}\right]=\mathbf{N}_{i} \Delta_{i} \mathbf{U}_{i} \quad(i=A, B, C),
$$

where

$$
\begin{gathered}
\mathbf{N}_{i}=\left[\begin{array}{cccccc}
0 & 0 & \pi_{i y} & 0 & \pi_{i z} & 0 \\
\pi_{i x} & 0 & 0 & 0 & 0 & \pi_{i z} \\
0 & \pi_{i x} & 0 & \pi_{i y} & 0 & 0
\end{array}\right], \\
\Delta_{i}=\operatorname{diag}\left(\left[\begin{array}{cccccc}
\Delta_{i x} & \Delta_{i x} & \Delta_{i y} & \Delta_{i y} & \Delta_{i z} & \Delta_{i z}
\end{array}\right]\right) ; \\
\mathbf{U}_{i}=\left[\begin{array}{cccccc}
0 & 0 & 0 & 1 & 0 & -1 \\
0 & -1 & 0 & 0 & 1 & 0 \\
1 & 0 & -1 & 0 & 0 & 0
\end{array}\right]^{T} ; \\
\Delta_{i j}=\frac{\varphi_{i j}}{\pi_{i j}}, \quad j=x, y, z .
\end{gathered}
$$

The parameters $\pi_{i j}$ are positive constants. If $\pi_{i j}$ are large enough, the inequalities $\Delta_{i}^{T} \Delta_{i} \leq \mathrm{I}$ and $\Delta_{i} \Delta_{i}^{T} \leq \mathrm{I}$ can be satisfied. For convenience, the uncertain measurement with misalignment errors can be rewritten as

$$
\mathbf{z}_{k}=\left(\mathbf{C}_{k}+\Delta \mathbf{C}_{k}\right) \mathbf{x}_{k}+\mathbf{v}_{k}
$$

where $\mathbf{v}_{k}$ is the zero mean Gaussian white-noise process with covariance $\mathbf{R}_{k}=\sigma_{s}^{2} \mathbf{I}_{9 \times 9}$,

$$
\begin{array}{cc}
\mathbf{z}_{k}=\left[\begin{array}{c}
\Delta \boldsymbol{\rho}_{A} \\
\Delta \boldsymbol{\rho}_{B} \\
\Delta \boldsymbol{\rho}_{C}
\end{array}\right], & \mathbf{C}_{k}=\left[\begin{array}{ll}
\mathbf{I}_{3 \times 3} & \mathbf{0}_{3 \times 3} \\
\mathbf{I}_{3 \times 3} & \mathbf{0}_{3 \times 3} \\
\mathbf{I}_{3 \times 3} & \mathbf{0}_{3 \times 3}
\end{array}\right], \\
\Delta \mathbf{C}_{k}=\mathbf{H}_{2, k} \mathbf{F}_{2, k} \mathbf{E}_{1, k}^{*}, & \mathbf{H}_{2, k}=\left[\begin{array}{lll}
\mathbf{N}_{A} & \\
& \mathbf{N}_{B} \\
& & \mathbf{N}_{C}
\end{array}\right], \\
\mathbf{F}_{2, k}=\left[\begin{array}{ccc}
\Delta_{A} & & \\
& \Delta_{B} & \\
& & \Delta_{C}
\end{array}\right], & \mathbf{E}_{1, k}^{*}=\left[\begin{array}{ll}
\mathbf{U}_{A} & \mathbf{0}_{6 \times 3} \\
\mathbf{U}_{B} & \mathbf{0}_{6 \times 3} \\
\mathbf{U}_{C} & \mathbf{0}_{6 \times 3}
\end{array}\right] .
\end{array}
$$

Since the attitude information of star sensors is given by the complicated data processing and transmission, the output measurement is delayed. The delayed star sensor measurement is assumed as

$$
\mathbf{y}_{k}=\left(\mathbf{I}-\boldsymbol{\Gamma}_{k}\right) \mathbf{z}_{k}+\boldsymbol{\Gamma}_{k} \mathbf{z}_{k-1},
$$

where $\mathbf{y}_{k} \in \mathbf{R}^{m}$ is the true measurement output vector, $\boldsymbol{\Gamma}_{k}=\operatorname{diag}\left\{\mu_{k, 1}, \mu_{k, 2}, \ldots, \mu_{k, m}\right\}$ accounts for the different delay rates, and $\mu_{k, i} \in \mathbf{R}(i=1,2, \ldots, m)$ are independent random variables taking the values of 1 or 0 with

$$
\begin{gathered}
p\left(\mu_{k, i}=1\right)=E\left[\mu_{k, i}\right]=p_{k, i} \\
p\left(\mu_{k, i}=0\right)=1-E\left[\mu_{k, i}\right]=1-p_{k, i}
\end{gathered}
$$

where $p_{k, i} \in[0,1)$ is a known scalar.

Remark 1. The measurement errors of sensors are inevitable in real applications, and the gyro and star sensor are no exception. Though the literature [14] takes into consideration the misalignment error of star sensors, no attention is paid to the misalignment errors and scale factor errors of the gyro. However, they can lead to the uncertainty process model. As shown in (11), the uncertainty error matrix exists in the state matrix and noise matrix, which influences the design of robust filter. Besides, the sensor measurement signal transmission is susceptible to interference from the external environment and limited bandwidth of network, which makes the sensor measurement delay occur. In (22), the delayed star sensor measurement model is established. As discussed in the work [25], different delay rates are taken into account by introducing the diagonal matrix $\boldsymbol{\Gamma}_{k}$.

\section{Finite-Horizon Robust Kalman Filter for Attitude Estimation}

3.1. Problem Description. Considering the uncertain discretetime linear stochastic system with sensor delays

$$
\begin{gathered}
\mathbf{x}_{k+1}=\left(\mathbf{A}_{k}+\Delta \mathbf{A}_{k}\right) \mathbf{x}_{k}+\left(\mathbf{B}_{k}+\Delta \mathbf{B}_{k}\right) \mathbf{w}_{k} \\
\mathbf{z}_{k}=\left(\mathbf{C}_{k}+\Delta \mathbf{C}_{k}\right) \mathbf{x}_{k}+\mathbf{v}_{k} \\
\mathbf{y}_{k}=\left(\mathbf{I}-\boldsymbol{\Gamma}_{k}\right) \mathbf{z}_{k}+\boldsymbol{\Gamma}_{k} \mathbf{z}_{k-1},
\end{gathered}
$$

where $\mathbf{x}_{k} \in \mathbf{R}^{n}$ is the state vector, $\mathbf{z}_{k} \in \mathbf{R}^{m}$ is the measurement vector, $\mathbf{y}_{k} \in \mathbf{R}^{m}$ is the true measurement output vector, $\mathbf{w}_{k}$ and $\mathbf{v}_{k}$ are uncorrelated process and measurement Gaussian noises with zero means and covariance $\mathbf{Q}_{k}$ and covariance $\mathbf{R}_{k}$, and $\mathbf{A}_{k}, \mathbf{B}_{k}$, and $\mathbf{C}_{k}$ are known matrices with appropriate dimensions. The matrices $\Delta \mathbf{A}_{k}, \Delta \mathbf{B}_{k}$, and $\Delta \mathbf{C}_{k}$ represent uncertainties in the state, process noise, and output matrices, which have the following form:

$$
\begin{gathered}
\Delta \mathbf{A}_{k}=\mathbf{H}_{1, k} \mathbf{F}_{1, k} \mathbf{E}_{1, k}, \quad \Delta \mathbf{B}_{k}=\mathbf{H}_{1, k} \mathbf{F}_{1, k} \mathbf{E}_{2, k}, \\
\Delta \mathbf{C}_{k}=\mathbf{H}_{2, k} \mathbf{F}_{2, k} \mathbf{E}_{1, k}^{*},
\end{gathered}
$$

where $\mathbf{H}_{1, k}, \mathbf{E}_{1, k}, \mathbf{E}_{2, k}, \mathbf{H}_{2, k}$, and $\mathbf{E}_{1, k}^{*}$ are known matrices with appropriate dimensions and $\mathbf{F}_{1, k}$ and $\mathbf{F}_{2, k}$ are the normbounded uncertainties satisfying $\mathbf{F}_{1, k} \mathbf{F}_{1, k}^{T} \leq \mathbf{I}$ and $\mathbf{F}_{2, k} \mathbf{F}_{2, k}^{T} \leq \mathbf{I}$. 
Due to the delayed measurement model, we need to obtain a concise model for convenience. By defining

$$
\begin{aligned}
& \mathbf{X}_{k}=\left[\begin{array}{c}
\mathbf{x}_{k} \\
\mathbf{x}_{k-1}
\end{array}\right], \quad \overline{\mathbf{A}}_{k}=\left[\begin{array}{cc}
\mathbf{A}_{k} & \mathbf{0} \\
\mathbf{0} & \mathbf{A}_{k-1}
\end{array}\right], \\
& \overline{\mathbf{B}}_{k}=\left[\begin{array}{cc}
\mathbf{B}_{k} & \mathbf{0} \\
\mathbf{0} & \mathbf{B}_{k-1}
\end{array}\right], \\
& \Delta \overline{\mathbf{A}}_{k}=\overline{\mathbf{H}}_{1, k} \overline{\mathbf{F}}_{1, k} \overline{\mathbf{E}}_{1, k}, \quad \Delta \overline{\mathbf{B}}_{k}=\overline{\mathbf{H}}_{1, k} \overline{\mathbf{F}}_{1, k} \overline{\mathbf{E}}_{2, k}, \\
& \overline{\mathbf{H}}_{1, k}=\left[\begin{array}{cc}
\mathbf{H}_{1, k} & \mathbf{0} \\
\mathbf{0} & \mathbf{H}_{1, k-1}
\end{array}\right], \quad \overline{\mathbf{F}}_{1, k}=\left[\begin{array}{cc}
\mathbf{F}_{1, k} & \mathbf{0} \\
\mathbf{0} & \mathbf{F}_{1, k-1}
\end{array}\right], \\
& \overline{\mathbf{E}}_{1, k}=\left[\begin{array}{cc}
\mathbf{E}_{1, k} & \mathbf{0} \\
\mathbf{0} & \mathbf{E}_{1, k-1}
\end{array}\right], \quad \overline{\mathbf{E}}_{2, k}=\left[\begin{array}{cc}
\mathbf{E}_{2, k} & \mathbf{0} \\
\mathbf{0} & \mathbf{E}_{2, k-1}
\end{array}\right], \\
& \overline{\mathbf{C}}_{k}=\left[\begin{array}{cc}
\mathbf{C}_{k} & \mathbf{0} \\
\mathbf{0} & \mathbf{C}_{k-1}
\end{array}\right], \quad \Delta \overline{\mathbf{C}}_{k}=\overline{\mathbf{H}}_{2, k} \overline{\mathbf{F}}_{2, k} \overline{\mathbf{E}}_{1, k}^{*}, \\
& \overline{\mathbf{H}}_{2, k}=\left[\begin{array}{cc}
\mathbf{H}_{2, k} & \mathbf{0} \\
\mathbf{0} & \mathbf{H}_{2, k-1}
\end{array}\right], \quad \overline{\mathbf{F}}_{2, k}=\left[\begin{array}{cc}
\mathbf{F}_{2, k} & \mathbf{0} \\
\mathbf{0} & \mathbf{F}_{2, k-1}
\end{array}\right], \\
& \overline{\mathbf{E}}_{1, k}^{*}=\left[\begin{array}{cc}
\mathbf{E}_{1, k}^{*} & \mathbf{0} \\
\mathbf{0} & \mathbf{E}_{1, k-1}^{*}
\end{array}\right], \quad \overline{\mathbf{w}}_{k}=\left[\begin{array}{c}
\mathbf{w}_{k} \\
\mathbf{w}_{k-1}
\end{array}\right], \\
& \overline{\mathbf{v}}_{k}=\left[\begin{array}{c}
\mathbf{v}_{k} \\
\mathbf{v}_{k-1}
\end{array}\right], \\
& \mathbf{Y}_{k}=\left[\begin{array}{ll}
\mathbf{I}-\mathbf{\Gamma}_{k} & \boldsymbol{\Gamma}_{k}
\end{array}\right],
\end{aligned}
$$

we have the following form:

$$
\begin{gathered}
\mathbf{X}_{k+1}=\left(\overline{\mathbf{A}}_{k}+\Delta \overline{\mathbf{A}}_{k}\right) \mathbf{X}_{k}+\left(\overline{\mathbf{B}}_{k}+\Delta \overline{\mathbf{B}}_{k}\right) \overline{\mathbf{w}}_{k} \\
\mathbf{y}_{k}=\mathbf{Y}_{k}\left[\left(\overline{\mathbf{C}}_{k}+\Delta \overline{\mathbf{C}}_{k}\right) \mathbf{X}_{k}+\overline{\mathbf{v}}_{k}\right],
\end{gathered}
$$

where it is known that

$$
\begin{gathered}
E\left[\overline{\mathbf{w}}_{k} \overline{\mathbf{w}}_{k}^{T}\right]=\overline{\mathbf{Q}}_{k}=\left[\begin{array}{cc}
\mathbf{Q}_{k} & \mathbf{0} \\
\mathbf{0} & \mathbf{Q}_{k-1}
\end{array}\right], \\
E\left[\overline{\mathbf{v}}_{k} \overline{\mathbf{v}}_{k}^{T}\right]=\overline{\mathbf{R}}_{k}=\left[\begin{array}{cc}
\mathbf{R}_{k} & \mathbf{0} \\
\mathbf{0} & \mathbf{R}_{k-1}
\end{array}\right] .
\end{gathered}
$$

According to the definition, $\mathbf{\Upsilon}_{k}$ can be expressed as

$$
\begin{gathered}
\overline{\mathbf{\Upsilon}}_{k}=E\left[\mathbf{\Upsilon}_{k}\right]=\left[\begin{array}{lll}
\mathbf{I}-\overline{\boldsymbol{\Gamma}}_{k} & \overline{\boldsymbol{\Gamma}}_{k}
\end{array}\right] \\
\widetilde{\mathbf{\Upsilon}}_{k}=\mathbf{\Upsilon}_{k}-\overline{\mathbf{\Upsilon}}_{k}=\left[\begin{array}{ll}
\overline{\boldsymbol{\Gamma}}_{k}-\boldsymbol{\Gamma}_{k} & \boldsymbol{\Gamma}_{k}-\overline{\boldsymbol{\Gamma}}_{k}
\end{array}\right],
\end{gathered}
$$

where $\overline{\boldsymbol{\Gamma}}_{k}=\operatorname{diag}\left\{p_{k, 1}, p_{k, 2}, \ldots, p_{k, m}\right\}$.

For the uncertain system (27), a required filter form is assumed as

$$
\widehat{\mathbf{X}}_{k+1}=\mathbf{A}_{o} \widehat{\mathbf{X}}_{k}+\mathbf{K}_{o}\left(\mathbf{y}_{k}-\overline{\mathbf{Y}}_{k} \overline{\mathbf{C}}_{k} \widehat{\mathbf{X}}_{k}\right),
$$

where $\widehat{\mathbf{X}}_{k}$ is the state estimation value with $\widehat{\mathbf{X}}_{0}=\left[\begin{array}{ll}\mathbf{x}_{0}^{T} & 0\end{array}\right]^{T}$ and $\mathbf{A}_{o}$ and $\mathbf{K}_{o}$ are the filter parameters to be determined. According to the above analysis, the robust filtering problem for delayed uncertain system (24) can be converted to the robust filter design problem for uncertain system (27). Therefore, our aim is to find an upper bound on the estimation error covariance and design a finite-horizon robust filter for (30) to minimize the upper bound.
Remark 2. Compared with the literature $[15,16]$, it is obvious that the designed robust Kalman filter does not apply to the case that the measurement delay appears in the system. Meanwhile, the definition of the uncertainty matrices $\Delta \mathbf{A}_{k}$ and $\Delta \mathbf{C}_{k}$ is different from the definition of the corresponding matrices in $[15,16]$. So, in order to facilitate the robust filter design, we need to utilize the state augmentation method to obtain a new uncertain system in (27).

3.2. Upper Bound of the Estimation Error Covariance. Because there are uncertain and delay rate terms for the system (27), it is difficult to obtain the true estimation error covariance. Our objective is to find the upper bound $\bar{\Xi}_{k}$, where

$$
E\left[\left(\mathbf{X}_{k}-\widehat{\mathbf{X}}_{k}\right)\left(\mathbf{X}_{k}-\widehat{\mathbf{X}}_{k}\right)^{T}\right] \leq \bar{\Xi}_{k}
$$

Considering the system (27) and the filter structure (30), we define an augmented state $\boldsymbol{\eta}_{k}=\left[\begin{array}{ll}\mathbf{X}_{k}^{T} & \widehat{\mathbf{X}}_{k}^{T}\end{array}\right]^{T}$. Then, the augmented state-space model is expressed as

$$
\begin{aligned}
\boldsymbol{\eta}_{k+1}= & \left(\widehat{\mathbf{A}}_{k}+\widehat{\mathbf{H}}_{1, k} \widehat{\mathbf{F}}_{1, k} \widehat{\mathbf{E}}_{1, k}\right) \boldsymbol{\eta}_{k}+\widehat{\mathbf{A}}_{1, k} \boldsymbol{\eta}_{k} \\
& +\left(\widehat{\mathbf{B}}_{k}+\widehat{\mathbf{H}}_{2, k} \widehat{\mathbf{F}}_{2, k} \widehat{\mathbf{E}}_{2, k}\right) \widehat{\mathbf{w}}_{k}+\widehat{\mathbf{B}}_{1, k} \widehat{\mathbf{w}}_{k},
\end{aligned}
$$

where

$$
\begin{aligned}
& \widehat{\mathbf{A}}_{k}=\left[\begin{array}{cc}
\overline{\mathbf{A}}_{k} & \mathbf{0} \\
\mathbf{K}_{o} \overline{\mathbf{Y}}_{k} \overline{\mathbf{C}}_{k} & \mathbf{A}_{o}-\mathbf{K}_{o} \overline{\mathbf{Y}}_{k} \overline{\mathbf{C}}_{k}
\end{array}\right], \\
& \widehat{\mathbf{H}}_{1, k}=\left[\begin{array}{cc}
\overline{\mathbf{H}}_{1, k} & \mathbf{0} \\
\mathbf{0} & \mathbf{K}_{o} \overline{\mathbf{\Upsilon}}_{k} \overline{\mathbf{H}}_{2, k}
\end{array}\right] \text {, } \\
& \widehat{\mathbf{E}}_{1, k}=\left[\begin{array}{cc}
\overline{\mathbf{E}}_{1, k} & \mathbf{0} \\
\overline{\mathbf{E}}_{1, k}^{*} & \mathbf{0}
\end{array}\right], \quad \widehat{\mathbf{F}}_{1, k}=\left[\begin{array}{cc}
\overline{\mathbf{F}}_{1, k} & \mathbf{0} \\
\mathbf{0} & \overline{\mathbf{F}}_{2, k}
\end{array}\right] \text {, }
\end{aligned}
$$

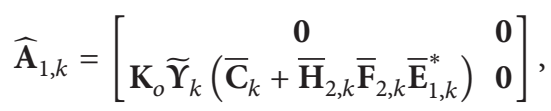

$$
\begin{aligned}
& \widehat{\mathbf{B}}_{k}=\left[\begin{array}{cc}
\overline{\mathbf{B}}_{k} & \mathbf{0} \\
\mathbf{0} & \mathbf{K}_{o} \overline{\mathbf{Y}}_{k}
\end{array}\right], \\
& \widehat{\mathbf{H}}_{2, k}=\left[\begin{array}{cc}
\overline{\mathbf{H}}_{1, k} & \mathbf{0} \\
\mathbf{0} & \mathbf{0}
\end{array}\right], \quad \widehat{\mathbf{F}}_{2, k}=\left[\begin{array}{cc}
\overline{\mathbf{F}}_{1, k} & \mathbf{0} \\
\mathbf{0} & \mathbf{0}
\end{array}\right] \text {, } \\
& \widehat{\mathbf{E}}_{2, k}=\left[\begin{array}{cc}
\overline{\mathbf{E}}_{2, k} & \mathbf{0} \\
\mathbf{0} & \mathbf{0}
\end{array}\right] \text {, } \\
& \widehat{\mathbf{B}}_{1, k}=\left[\begin{array}{cc}
\mathbf{0} & \mathbf{0} \\
\mathbf{0} & \mathbf{K}_{o} \widetilde{\mathbf{Y}}_{k}
\end{array}\right], \quad \widehat{\mathbf{w}}_{k}=\left[\begin{array}{c}
\overline{\mathbf{w}}_{k} \\
\overline{\mathbf{v}}_{k}
\end{array}\right] \text {. }
\end{aligned}
$$

The state covariance matrix of $\boldsymbol{\eta}_{k}$ in augmented system (32) is denoted as

$$
\boldsymbol{\Xi}_{k}=E\left[\boldsymbol{\eta}_{k} \boldsymbol{\eta}_{k}^{T}\right]=\left[\begin{array}{ll}
\boldsymbol{\Xi}_{11, k} & \boldsymbol{\Xi}_{12, k} \\
\boldsymbol{\Xi}_{12, k}^{T} & \boldsymbol{\Xi}_{22, k}
\end{array}\right]
$$


So, the evolution equation can be expressed as

$$
\begin{aligned}
\boldsymbol{\Xi}_{k+1}= & \left(\widehat{\mathbf{A}}_{k}+\widehat{\mathbf{H}}_{1, k} \widehat{\mathbf{F}}_{1, k} \widehat{\mathbf{E}}_{1, k}\right) \boldsymbol{\Xi}_{k}\left(\widehat{\mathbf{A}}_{k}+\widehat{\mathbf{H}}_{1, k} \widehat{\mathbf{F}}_{1, k} \widehat{\mathbf{E}}_{1, k}\right)^{T}+\boldsymbol{\Psi}_{1} \\
& +\left(\widehat{\mathbf{B}}_{k}+\widehat{\mathbf{H}}_{2, k} \widehat{\mathbf{F}}_{2, k} \widehat{\mathbf{E}}_{2, k}\right) \mathbf{W}_{k}\left(\widehat{\mathbf{B}}_{k}+\widehat{\mathbf{H}}_{2, k} \widehat{\mathbf{F}}_{2, k} \widehat{\mathbf{E}}_{2, k}\right)^{T}+\Psi_{2},
\end{aligned}
$$

where

$$
\begin{aligned}
& \mathbf{W}_{k}=\left[\begin{array}{cc}
\overline{\mathbf{Q}}_{k} & \mathbf{0} \\
\mathbf{0} & \overline{\mathbf{R}}_{k}
\end{array}\right], \quad \widehat{\mathbf{F}}_{1, k} \widehat{\mathbf{F}}_{1, k}^{T} \leq \mathbf{I}, \quad \widehat{\mathbf{F}}_{2, k} \widehat{\mathbf{F}}_{2, k}^{T} \leq \mathbf{I} \\
& \boldsymbol{\Psi}_{1}=E\left[\widehat{\mathbf{A}}_{1, k} \boldsymbol{\eta}_{k} \boldsymbol{\eta}_{k}^{T} \widehat{\mathbf{A}}_{1, k}^{T}\right]
\end{aligned}
$$

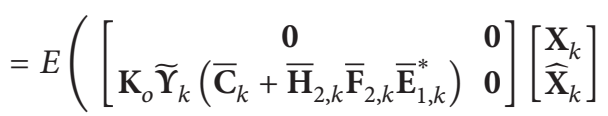

$$
\begin{aligned}
& \left.\times\left[\begin{array}{l}
\mathbf{X}_{k} \\
\widehat{\mathbf{X}}_{k}
\end{array}\right]^{T}\left[\mathbf{K}_{o} \widetilde{\mathbf{Y}}_{k}\left(\overline{\mathbf{C}}_{k}+\overline{\mathbf{H}}_{2, k} \overline{\mathbf{F}}_{2, k} \overline{\mathbf{E}}_{1, k}^{*}\right) \quad \mathbf{0}\right]^{T}\right)
\end{aligned}
$$

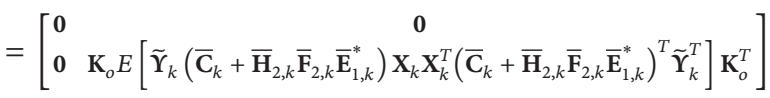

$$
\begin{aligned}
& \boldsymbol{\Psi}_{2}=E\left[\widehat{\mathbf{B}}_{1, k} \widehat{\mathbf{w}}_{k} \widehat{\mathbf{w}}_{k}^{T} \widehat{\mathbf{B}}_{1, k}^{T}\right] \\
& =E\left(\left[\begin{array}{cc}
\mathbf{0} & \mathbf{0} \\
\mathbf{0} & \mathbf{K}_{o} \widetilde{\mathbf{Y}}_{k}
\end{array}\right]\left[\begin{array}{c}
\overline{\mathbf{w}}_{k} \\
\overline{\mathbf{v}}_{k}
\end{array}\right]\left[\begin{array}{c}
\overline{\mathbf{w}}_{k} \\
\overline{\mathbf{v}}_{k}
\end{array}\right]^{T}\left[\begin{array}{cc}
\mathbf{0} & \mathbf{0} \\
\mathbf{0} & \mathbf{K}_{o} \widetilde{\mathbf{Y}}_{k}
\end{array}\right]^{T}\right) \\
& =\left[\begin{array}{cc}
\mathbf{0} & \mathbf{0} \\
\mathbf{0} & \mathbf{K}_{o} E\left[\widetilde{\mathbf{Y}}_{k} \overline{\mathbf{v}}_{k} \overline{\mathbf{v}}_{k}^{T} \widetilde{\mathbf{Y}}_{k}^{T}\right] \mathbf{K}_{o}^{T}
\end{array}\right] .
\end{aligned}
$$

In order to obtain the upper bound of the error covariance in (35), the following two lemmas are employed.

Lemma 3 (see [27]). Given matrices $\mathbf{A}, \mathbf{H}, \mathbf{E}$, and $\mathbf{F}$ with compatible dimensions such that $\mathbf{F F}^{T} \leq \mathbf{I}$, let $\mathbf{X}$ be a symmetric positive definite matrix and let $\gamma$ be an arbitrary positive constant such that

$$
\gamma^{-1} \mathbf{I}-\mathbf{E X E}^{T}>0 \text {. }
$$

Then, the following matrix inequality holds:

$$
\begin{aligned}
& (\mathbf{A}+\mathbf{H F E}) \mathbf{X}(\mathbf{A}+\mathbf{H F E})^{T} \\
& \quad \leq \mathbf{A}\left(\mathbf{X}^{-1}-\gamma \mathbf{E}^{T} \mathbf{E}\right)^{-1} \mathbf{A}^{T}+\gamma^{-1} \mathbf{H} \mathbf{H}^{T} .
\end{aligned}
$$

Lemma 4 (see [28]). Let $\mathbf{A}=\left[a_{i j}\right]_{n \times n}$ be a real matrix and let $\mathbf{B}=\operatorname{diag}\left(b_{1}, b_{2}, \ldots, b_{n}\right)$ be a diagonal random matrix. Then,

$$
E\left\{\mathbf{B A B}^{T}\right\}=\left[\begin{array}{cccc}
E\left\{b_{1}^{2}\right\} & E\left\{b_{1} b_{2}\right\} & \cdots & E\left\{b_{1} b_{n}\right\} \\
E\left\{b_{2} b_{1}\right\} & E\left\{b_{2}^{2}\right\} & \cdots & E\left\{b_{2} b_{n}\right\} \\
\vdots & \vdots & \ddots & \vdots \\
E\left\{b_{n} b_{1}\right\} & E\left\{b_{n} b_{2}\right\} & \cdots & E\left\{b_{n}^{2}\right\}
\end{array}\right] \circ \mathbf{A},
$$

Then, the following conclusion can be given by making use of the two lemmas.

Theorem 5. If there exist three positive scalars $\lambda_{1}, \lambda_{2}$, and $\lambda_{3}$, such that

$$
\begin{gathered}
\lambda_{1}^{-1} \mathbf{I}-\widehat{\mathbf{E}}_{1, k} \boldsymbol{\Xi}_{k} \widehat{\mathbf{E}}_{1, k}^{T}>0 \\
\lambda_{2}^{-1} \mathbf{I}-\widehat{\mathbf{E}}_{2, k} \mathbf{W}_{k} \widehat{\mathbf{E}}_{2, k}^{T}>0 \\
\lambda_{3}^{-1} \mathbf{I}-\overline{\mathbf{E}}_{1, k}^{*} \boldsymbol{\Xi}_{11, k}\left(\overline{\mathbf{E}}_{1, k}^{*}\right)^{T}>0
\end{gathered}
$$

and there exists a symmetric positive-definite matrix $\widetilde{\Xi}_{k}$, such that

$$
\begin{aligned}
\widetilde{\boldsymbol{\Xi}}_{k+1}= & \widehat{\mathbf{A}}_{k}\left(\widetilde{\boldsymbol{\Xi}}_{k}^{-1}-\lambda_{1} \widehat{\mathbf{E}}_{1, k}^{T} \widehat{\mathbf{E}}_{1, k}\right)^{-1} \widehat{\mathbf{A}}_{k}^{T} \\
& +\lambda_{1}^{-1} \widehat{\mathbf{H}}_{1, k} \widehat{\mathbf{H}}_{1, k}^{T}+\lambda_{2}^{-1} \widehat{\mathbf{H}}_{2, k} \widehat{\mathbf{H}}_{2, k}^{T} \\
& +\widehat{\mathbf{B}}_{k}\left(\mathbf{W}_{k}^{-1}-\lambda_{2} \widehat{\mathbf{E}}_{2, k}^{T} \widehat{\mathbf{E}}_{2, k}\right)^{-1} \widehat{\mathbf{B}}_{k}^{T} \\
& +\left[\begin{array}{ll}
\mathbf{0} & \mathbf{0} \\
\mathbf{0} & \mathbf{K}_{o}\left(\boldsymbol{\Phi}_{1, k}+\boldsymbol{\Phi}_{2, k}\right) \mathbf{K}_{o}^{T}
\end{array}\right],
\end{aligned}
$$

where

$$
\begin{gathered}
\boldsymbol{\Phi}_{1, k}=\breve{\boldsymbol{\Gamma}}_{k} \circ\left\{\overline { \mathbf { H } } \left[\overline { \mathbf { C } } _ { k } \left(\widetilde{\boldsymbol{\Xi}}_{11, k}+\widetilde{\boldsymbol{\Xi}}_{11, k}\left(\overline{\mathbf{E}}_{1, k}^{*}\right)^{T}\right.\right.\right. \\
\times\left(\lambda_{3}^{-1} \mathbf{I}-\overline{\mathbf{E}}_{1, k}^{*} \widetilde{\boldsymbol{\Xi}}_{11, k}\left(\overline{\mathbf{E}}_{1, k}^{*}\right)^{T}\right)^{-1} \\
\left.\left.\left.\times \overline{\mathbf{E}}_{1, k}^{*} \widetilde{\boldsymbol{\Xi}}_{11, k}\right) \overline{\mathbf{C}}_{k}^{T}+\lambda_{3}^{-1} \overline{\mathbf{H}}_{2, k} \overline{\mathbf{H}}_{2, k}^{T}\right] \overline{\mathbf{H}}^{T}\right\} \\
\boldsymbol{\Phi}_{2, k}=\breve{\boldsymbol{\Gamma}}_{k} \circ\left(\overline{\mathbf{H R}}_{k} \overline{\mathbf{H}}^{T}\right), \quad \overline{\mathbf{H}}=\left[\mathbf{I}_{m}-\mathbf{I}_{m}\right] \\
\breve{\mathbf{\Gamma}}_{k}=\operatorname{diag}\left\{p_{k, 1}\left(1-p_{k, 1}\right), p_{k, 2}\left(1-p_{k, 2}\right), \ldots,\right. \\
\left.p_{k, m}\left(1-p_{k, m}\right)\right\}
\end{gathered}
$$

with initial value $\widetilde{\boldsymbol{\Xi}}_{0}=\operatorname{diag}\left\{\mathbf{U}_{0}, \mathbf{0}\right\}$, then $\boldsymbol{\Xi}_{k} \leq \widetilde{\boldsymbol{\Xi}}_{k}$ for $0 \leq k \leq$ N.

Proof. According to Lemma 4, we have

$$
\begin{gathered}
E\left[\widetilde{\mathbf{Y}}_{k} \overline{\mathbf{v}}_{k} \overline{\mathbf{v}}_{k}^{T} \widetilde{\mathbf{Y}}_{k}^{T}\right]=\breve{\mathbf{\Gamma}}_{k} \circ\left(\overline{\mathbf{H R}}_{k} \overline{\mathbf{H}}^{T}\right) \\
E\left[\widetilde{\mathbf{Y}}_{k}\left(\overline{\mathbf{C}}_{k}+\overline{\mathbf{H}}_{2, k} \overline{\mathbf{F}}_{2, k} \overline{\mathbf{E}}_{1, k}^{*}\right) \mathbf{X}_{k} \mathbf{X}_{k}^{T}\left(\overline{\mathbf{C}}_{k}+\overline{\mathbf{H}}_{2, k} \overline{\mathbf{F}}_{2, k} \overline{\mathbf{E}}_{1, k}^{*}\right)^{T} \widetilde{\mathbf{Y}}_{k}^{T}\right] \\
=\breve{\mathbf{\Gamma}}_{k} \circ\left\{\overline { \mathbf { H } } \left[\left(\overline{\mathbf{C}}_{k}+\overline{\mathbf{H}}_{2, k} \overline{\mathbf{F}}_{2, k} \overline{\mathbf{E}}_{1, k}^{*}\right) \mathbf{\Xi}_{11, k}\right.\right. \\
\left.\left.\times\left(\overline{\mathbf{C}}_{k}+\overline{\mathbf{H}}_{2, k} \overline{\mathbf{F}}_{2, k} \overline{\mathbf{E}}_{1, k}^{*}\right)^{T}\right] \overline{\mathbf{H}}^{T}\right\} .
\end{gathered}
$$

where $\circ$ is the Hadamard product. 
Since the assumptions in (40) hold, using Lemma 3, we can get

$$
\begin{aligned}
\boldsymbol{\Xi}_{k+1} \leq & \widehat{\mathbf{A}}_{k}\left(\boldsymbol{\Xi}_{k}^{-1}-\lambda_{1} \widehat{\mathbf{E}}_{1, k}^{T} \widehat{\mathbf{E}}_{1, k}\right)^{-1} \widehat{\mathbf{A}}_{k}^{T} \\
& +\lambda_{1}^{-1} \widehat{\mathbf{H}}_{1, k} \widehat{\mathbf{H}}_{1, k}^{T}+\lambda_{2}^{-1} \widehat{\mathbf{H}}_{2, k} \widehat{\mathbf{H}}_{2, k}^{T} \\
& +\widehat{\mathbf{B}}_{k}\left(\mathbf{W}_{k}^{-1}-\lambda_{2} \widehat{\mathbf{E}}_{2, k}^{T} \widehat{\mathbf{E}}_{2, k}\right)^{-1} \widehat{\mathbf{B}}_{k}^{T} \\
& +\left[\begin{array}{lc}
\mathbf{0} & \mathbf{0} \\
\mathbf{0} & \mathbf{K}_{o}\left(\overline{\boldsymbol{\Phi}}_{1, k}+\boldsymbol{\Phi}_{2, k}\right) \mathbf{K}_{o}^{T}
\end{array}\right],
\end{aligned}
$$

where

$$
\begin{gathered}
\overline{\boldsymbol{\Phi}}_{1, k}=\breve{\mathbf{\Gamma}}_{k} \circ\left\{\overline { \mathbf { H } } \left[\overline{\mathbf{C}}_{k}\left(\mathbf{\Xi}_{11, k}^{-1}-\lambda_{3}\left(\overline{\mathbf{E}}_{1, k}^{*}\right)^{T} \overline{\mathbf{E}}_{1, k}^{*}\right)^{-1} \overline{\mathbf{C}}_{k}^{T}\right.\right. \\
\left.\left.+\lambda_{3}^{-1} \overline{\mathbf{H}}_{2, k} \overline{\mathbf{H}}_{2, k}^{T}\right] \overline{\mathbf{H}}^{T}\right\} \\
\boldsymbol{\Phi}_{2, k}=\breve{\boldsymbol{\Gamma}}_{k} \circ\left(\overline{\mathbf{H R}}_{k} \overline{\mathbf{H}}^{T}\right), \quad \overline{\mathbf{H}}=\left[\mathbf{I}_{m}-\mathbf{I}_{m}\right] \\
\breve{\mathbf{\Gamma}}_{k}=\operatorname{diag}\left\{p_{k, 1}\left(1-p_{k, 1}\right), p_{k, 2}\left(1-p_{k, 2}\right), \ldots,\right. \\
\left.p_{k, m}\left(1-p_{k, m}\right)\right\} .
\end{gathered}
$$

Then, when $k=0$, we have $\boldsymbol{\Xi}_{0}=\widetilde{\boldsymbol{\Xi}}_{0}=\operatorname{diag}\left\{\mathbf{U}_{0}, \mathbf{0}\right\}$.

When $k=n$, suppose that $\Xi_{n} \leq \widetilde{\Xi}_{n}$.

When $k=n+1$, comparing (41) with (44), it is easy to obtain that $\Xi_{n+1} \leq \widetilde{\Xi}_{n+1}$. According to the mathematical induction, the proof is complete.

Assume that $\overline{\boldsymbol{\Xi}}_{k}=\left[\begin{array}{ll}\mathbf{I} & -\mathbf{I}\end{array}\right] \widetilde{\mathbf{\Xi}}_{k}[\mathbf{I}-\mathbf{I}]^{T}$; then

$$
E\left[\left(\mathbf{X}_{k}-\widehat{\mathbf{X}}_{k}\right)\left(\mathbf{X}_{k}-\widehat{\mathbf{X}}_{k}\right)^{T}\right] \leq \bar{\Xi}_{k}
$$

Therefore, for the upper bound $\overline{\boldsymbol{\Xi}}_{k}$, we need to choose the filter parameters $\mathbf{A}_{o}$ and $\mathbf{K}_{o}$ to obtain an optimized upper bound. In the next part, we will find the value of $\overline{\boldsymbol{\Xi}}_{k}$ and design the finite-horizon robust Kalman filter to minimize the upper bound.

3.3. Finite-Horizon Robust Kalman Filter Design. In order to obtain a solution to the above question and design the robust filter, the main result is presented in the following theorem.

Theorem 6. Assume that the positive scalars $\lambda_{1}, \lambda_{2}$, and $\lambda_{3}$ fulfill the assumptions in (40); then the upper bound $\widetilde{\Xi}_{k}$ can be expressed as

$$
\widetilde{\Xi}_{n}=\left[\begin{array}{ll}
\widetilde{\Xi}_{11, n} & \widetilde{\Xi}_{12, n} \\
\widetilde{\Xi}_{12, n}^{T} & \widetilde{\Xi}_{22, n}
\end{array}\right]=\left[\begin{array}{ll}
\widetilde{\Xi}_{11, n} & \widetilde{\Xi}_{22, n} \\
\widetilde{\Xi}_{22, n} & \widetilde{\Xi}_{22, n}
\end{array}\right], \quad n \in[0, N] .
$$

If the filter parameters $\mathbf{A}_{o}$ and $\mathbf{K}_{o}$ can be written as

$$
\begin{gathered}
\mathbf{A}_{o}=\overline{\mathbf{A}}_{k}+\left(\overline{\mathbf{A}}_{k}-\mathbf{K}_{o} \overline{\mathbf{Y}}_{k} \overline{\mathbf{C}}_{k}\right) \bar{\Xi}_{k} \mathbf{G}_{k}^{T}\left(\lambda_{1}^{-1} \mathbf{I}-\mathbf{G}_{k} \overline{\mathbf{\Xi}}_{k} \mathbf{G}_{k}^{T}\right)^{-1} \mathbf{G}_{k} \\
\mathbf{K}_{o}=\overline{\mathbf{A}}_{k} \mathbf{S}_{k} \overline{\mathbf{C}}_{k}^{T} \overline{\mathbf{Y}}_{k}^{T}\left[\overline{\mathbf{Y}}_{k} \overline{\mathbf{C}}_{k} \mathbf{S}_{k} \overline{\mathbf{C}}_{k}^{T} \overline{\mathbf{Y}}_{k}^{T}+\overline{\mathbf{Y}}_{k}\left(\lambda_{1}^{-1} \overline{\mathbf{H}}_{2, k} \overline{\mathbf{H}}_{2, k}^{T}+\overline{\mathbf{R}}_{k}\right)\right. \\
\left.\times \overline{\mathbf{Y}}_{k}^{T}+\boldsymbol{\Phi}_{1, k}+\boldsymbol{\Phi}_{2, k}\right]^{-1},
\end{gathered}
$$

where

$$
\begin{gathered}
\overline{\mathbf{E}}_{1, k}^{T} \overline{\mathbf{E}}_{1, k}+\left(\overline{\mathbf{E}}_{1, k}^{*}\right)^{T} \overline{\mathbf{E}}_{1, k}^{*}=\mathbf{G}_{k}^{T} \mathbf{G}_{k}, \\
\mathbf{S}_{k}=\overline{\boldsymbol{\Xi}}_{k}+\overline{\boldsymbol{\Xi}}_{k} \mathbf{G}_{k}^{T}\left(\lambda_{1}^{-1} \mathbf{I}-\mathbf{G}_{k} \overline{\mathbf{\Xi}}_{k} \mathbf{G}_{k}^{T}\right)^{-1} \mathbf{G}_{k} \overline{\mathbf{\Xi}}_{k},
\end{gathered}
$$

then $\operatorname{tr}\left(\overline{\boldsymbol{\Xi}}_{k}\right)$ is minimized. So, the state covariance can be obtained as

$$
\begin{aligned}
\widetilde{\Xi}_{11, k+1}= & \overline{\mathbf{A}}_{k}\left(\widetilde{\mathbf{\Xi}}_{11, k}+\widetilde{\mathbf{\Xi}}_{11, k} \mathbf{G}_{k}^{T}\left(\lambda_{1}^{-1} \mathbf{I}-\mathbf{G}_{k} \widetilde{\mathbf{\Xi}}_{11, k} \mathbf{G}_{k}^{T}\right)^{-1}\right. \\
& \left.\quad \times \mathbf{G}_{k} \widetilde{\Xi}_{11, k}\right) \overline{\mathbf{A}}_{k}^{T}+\overline{\mathbf{B}}_{k}\left(\overline{\mathbf{Q}}_{k}^{-1}-\lambda_{2} \overline{\mathbf{E}}_{2, k}^{T} \overline{\mathbf{E}}_{2, k}\right)^{-1} \overline{\mathbf{B}}_{k}^{T} \\
& +\left(\lambda_{1}^{-1}+\lambda_{2}^{-1}\right) \overline{\mathbf{H}}_{1, k} \overline{\mathbf{H}}_{1, k}^{T}
\end{aligned}
$$

and the estimation error covariance can be given as

$$
\begin{aligned}
\bar{\Xi}_{k+1}= & \overline{\mathbf{A}}_{k} \mathbf{S}_{k} \overline{\mathbf{A}}_{k}^{T}+\overline{\mathbf{B}}_{k}\left(\overline{\mathbf{Q}}_{k}^{-1}-\lambda_{2} \overline{\mathbf{E}}_{2, k}^{T} \overline{\mathbf{E}}_{2, k}\right)^{-1} \overline{\mathbf{B}}_{k}^{T}-\overline{\mathbf{A}}_{k} \mathbf{S}_{k} \overline{\mathbf{C}}_{k}^{T} \overline{\mathbf{Y}}_{k}^{T} \\
& \times\left[\overline{\mathbf{Y}}_{k} \overline{\mathbf{C}}_{k} \mathbf{S}_{k} \overline{\mathbf{C}}_{k}^{T} \overline{\mathbf{Y}}_{k}^{T}+\overline{\mathbf{Y}}_{k}\left(\lambda_{1}^{-1} \overline{\mathbf{H}}_{2, k} \overline{\mathbf{H}}_{2, k}^{T}+\overline{\mathbf{R}}_{k}\right) \overline{\mathbf{Y}}_{k}^{T}\right. \\
& \left.+\boldsymbol{\Phi}_{1, k}+\boldsymbol{\Phi}_{2, k}\right]^{-1} \overline{\mathbf{Y}}_{k} \overline{\mathbf{C}}_{k} \mathbf{S}_{k}^{T} \overline{\mathbf{A}}_{k}^{T} \\
& +\left(\lambda_{1}^{-1}+\lambda_{2}^{-1}\right) \overline{\mathbf{H}}_{1, k} \overline{\mathbf{H}}_{1, k}^{T} .
\end{aligned}
$$

Proof. when $n=0$, we have $\widetilde{\Xi}_{0}=\left[\begin{array}{ll}\widetilde{\Xi}_{11,0} & \widetilde{\Xi}_{22,0} \\ \widetilde{\Xi}_{22,0} & \widetilde{\Xi}_{22,0}\end{array}\right]=\left[\begin{array}{cc}\mathbf{U}_{0} & \mathbf{0} \\ \mathbf{0} & \mathbf{0}\end{array}\right]$. When $n=k$, assume that (47) is valid. When $n=k+1$, we will prove that (47) is still valid. From (47), suppose that the upper 
bound $\widetilde{\Xi}_{k+1}$ can be partitioned as $\left[\begin{array}{cc}\widetilde{\Xi}_{11, k+1} & \widetilde{\Xi}_{12, k+1} \\ \widetilde{\Xi}_{12, k+1}^{T} & \widetilde{\Xi}_{22, k+1}\end{array}\right]$. According to the definitions of (50), inserting $\widetilde{\Xi}_{k}$ into (41), we have

$$
\begin{aligned}
\widetilde{\Xi}_{11, k+1}= & \overline{\mathbf{A}}_{k} \widetilde{\Xi}_{11 c, k} \overline{\mathbf{A}}_{k}^{T}+\overline{\mathbf{B}}_{k}\left(\overline{\mathbf{Q}}_{k}^{-1}-\lambda_{2} \overline{\mathbf{E}}_{2, k}^{T} \overline{\mathbf{E}}_{2, k}\right)^{-1} \overline{\mathbf{B}}_{k}^{T} \\
& +\left(\lambda_{1}^{-1}+\lambda_{2}^{-1}\right) \overline{\mathbf{H}}_{1, k} \overline{\mathbf{H}}_{1, k}^{T}, \\
\widetilde{\Xi}_{12, k+1}= & \overline{\mathbf{A}}_{k} \widetilde{\Xi}_{11 c, k}\left(\mathbf{K}_{o} \overline{\mathbf{Y}}_{k} \overline{\mathbf{C}}_{k}\right)^{T}+\overline{\mathbf{A}}_{k} \widetilde{\Xi}_{12 c, k}\left(\mathbf{A}_{o}-\mathbf{K}_{o} \overline{\mathbf{Y}}_{k} \overline{\mathbf{C}}_{k}\right)^{T}, \\
\widetilde{\Xi}_{22, k+1}= & \mathbf{K}_{o} \overline{\mathbf{Y}}_{k} \overline{\mathbf{C}}_{k} \widetilde{\Xi}_{11 c, k}\left(\mathbf{K}_{o} \overline{\mathbf{Y}}_{k} \overline{\mathbf{C}}_{k}\right)^{T} \\
& +\left(\mathbf{A}_{o}-\mathbf{K}_{o} \overline{\mathbf{Y}}_{k} \overline{\mathbf{C}}_{k}\right) \widetilde{\Xi}_{12 c, k}^{T}\left(\mathbf{K}_{o} \overline{\mathbf{Y}}_{k} \overline{\mathbf{C}}_{k}\right)^{T} \\
& +\left(\mathbf{K}_{o} \overline{\mathbf{Y}}_{k} \overline{\mathbf{C}}_{k}\right) \widetilde{\Xi}_{12 c, k}\left(\mathbf{A}_{o}-\mathbf{K}_{o} \overline{\mathbf{Y}}_{k} \overline{\mathbf{C}}_{k}\right)^{T} \\
& +\left(\mathbf{A}_{o}-\mathbf{K}_{o} \overline{\mathbf{Y}}_{k} \overline{\mathbf{C}}_{k}\right) \widetilde{\boldsymbol{\Xi}}_{22 c, k}\left(\mathbf{A}_{o}-\mathbf{K}_{o} \overline{\mathbf{Y}}_{k} \overline{\mathbf{C}}_{k}\right)^{T} \\
& +\mathbf{K}_{o}\left[\overline{\mathbf{Y}}_{k}\left(\lambda_{1}^{-1} \overline{\mathbf{H}}_{2, k} \overline{\mathbf{H}}_{2, k}^{T}+\overline{\mathbf{R}}_{k}\right) \overline{\mathbf{Y}}_{k}^{T}\right. \\
& \left.+\boldsymbol{\Phi}_{1, k}+\boldsymbol{\Phi}_{2, k}\right] \mathbf{K}_{o}^{T},
\end{aligned}
$$

where

$$
\begin{gathered}
\mathbf{M}_{k}=\mathbf{G}_{k}^{T}\left(\lambda_{1}^{-1} \mathbf{I}-\mathbf{G}_{k} \widetilde{\Xi}_{11, k} \mathbf{G}_{k}^{T}\right)^{-1} \mathbf{G}_{k}, \\
\widetilde{\Xi}_{11 c, k}=\widetilde{\Xi}_{11, k}+\widetilde{\Xi}_{11, k} \mathbf{M}_{k} \widetilde{\Xi}_{11, k}, \\
\widetilde{\Xi}_{12 c, k}=\widetilde{\Xi}_{12, k}+\widetilde{\Xi}_{11, k} \mathbf{M}_{k} \widetilde{\Xi}_{12, k}, \\
\widetilde{\Xi}_{22 c, k}=\widetilde{\Xi}_{22, k}+\widetilde{\Xi}_{12, k}^{T} \mathbf{M}_{k} \widetilde{\Xi}_{12, k}, \\
\mathbf{S}_{k}=\widetilde{\Xi}_{11 c, k}-\widetilde{\Xi}_{12 c, k} \widetilde{\Xi}_{22 c, k}^{-1} \widetilde{\Xi}_{12 c, k}^{T} .
\end{gathered}
$$

Due to the fact that $\bar{\Xi}_{k}=\left[\begin{array}{ll}\mathbf{I} & -\mathbf{I}\end{array}\right] \widetilde{\boldsymbol{\Xi}}_{k}[\mathbf{I}-\mathbf{I}]^{T}$, using (54)(56), the required upper bound $\bar{\Xi}_{k+1}$ can be calculated as

$$
\begin{aligned}
\bar{\Xi}_{k+1}= & \widetilde{\Xi}_{11, k+1}-\widetilde{\Xi}_{12, k+1}-\widetilde{\Xi}_{12, k+1}^{T}+\widetilde{\Xi}_{22, k+1} \\
= & \left(\overline{\mathbf{A}}_{k}-\mathbf{K}_{o} \overline{\mathbf{Y}}_{k} \overline{\mathbf{C}}_{k}\right) \widetilde{\Xi}_{11 c, k}\left(\overline{\mathbf{A}}_{k}-\mathbf{K}_{o} \overline{\mathbf{Y}}_{k} \overline{\mathbf{C}}_{k}\right)^{T} \\
& -\left(\overline{\mathbf{A}}_{k}-\mathbf{K}_{o} \overline{\mathbf{Y}}_{k} \overline{\mathbf{C}}_{k}\right) \widetilde{\Xi}_{12 c, k}\left(\mathbf{A}_{o}-\mathbf{K}_{o} \overline{\mathbf{Y}}_{k} \overline{\mathbf{C}}_{k}\right)^{T} \\
& -\left(\mathbf{A}_{o}-\mathbf{K}_{o} \overline{\mathbf{Y}}_{k} \overline{\mathbf{C}}_{k}\right) \widetilde{\Xi}_{12 c, k}^{T}\left(\overline{\mathbf{A}}_{k}-\mathbf{K}_{o} \overline{\mathbf{Y}}_{k} \overline{\mathbf{C}}_{k}\right)^{T} \\
& +\left(\mathbf{A}_{o}-\mathbf{K}_{o} \overline{\mathbf{Y}}_{k} \overline{\mathbf{C}}_{k}\right) \widetilde{\Xi}_{22 c, k}\left(\mathbf{A}_{o}-\mathbf{K}_{o} \overline{\mathbf{Y}}_{k} \overline{\mathbf{C}}_{k}\right)^{T} \\
& +\overline{\mathbf{B}}_{k}\left(\overline{\mathbf{Q}}_{k}^{-1}-\lambda_{2} \overline{\mathbf{E}}_{2, k}^{T} \overline{\mathbf{E}}_{2, k}\right)^{-1} \overline{\mathbf{B}}_{k}^{T} \\
& +\left(\lambda_{1}^{-1}+\lambda_{2}^{-1}\right) \overline{\mathbf{H}}_{1, k} \overline{\mathbf{H}}_{1, k}^{T} \\
& +\mathbf{K}_{o}\left[\overline{\mathbf{Y}}_{k}\left(\lambda_{1}^{-1} \overline{\mathbf{H}}_{2, k} \overline{\mathbf{H}}_{2, k}^{T}+\overline{\mathbf{R}}_{k}\right) \overline{\mathbf{Y}}_{k}^{T}\right. \\
& \left.\quad+\boldsymbol{\Phi}_{1, k}+\boldsymbol{\Phi}_{2, k}\right] \mathbf{K}_{o}^{T} .
\end{aligned}
$$

Computing the first-order variation of (58) with respect to $\mathbf{A}_{o}$ and $\mathbf{K}_{o}$ and making them equal to zero, we have

$$
\begin{aligned}
& \frac{\partial \operatorname{tr}\left(\overline{\boldsymbol{\Xi}}_{k+1}\right)}{\partial \mathbf{A}_{o}}=-2\left(\overline{\mathbf{A}}_{k}-\mathbf{K}_{o} \overline{\mathbf{Y}}_{k} \overline{\mathbf{C}}_{k}\right) \widetilde{\boldsymbol{\Xi}}_{12 c, k} \\
& +2\left(\mathbf{A}_{o}-\mathbf{K}_{o} \overline{\mathbf{\Upsilon}}_{k} \overline{\mathbf{C}}_{k}\right) \widetilde{\boldsymbol{\Xi}}_{22 c, k}=0, \\
& \frac{\partial \operatorname{tr}\left(\overline{\boldsymbol{\Xi}}_{k+1}\right)}{\partial \mathbf{K}_{o}}=-2\left(\overline{\mathbf{A}}_{k}-\mathbf{K}_{o} \overline{\mathbf{Y}}_{k} \overline{\mathbf{C}}_{k}\right) \widetilde{\boldsymbol{\Xi}}_{11 c, k} \overline{\mathbf{C}}_{k}^{T} \overline{\mathbf{Y}}_{k}^{T} \\
& +2 \overline{\mathbf{A}}_{k} \widetilde{\Xi}_{12 c, k} \overline{\mathbf{C}}_{k}^{T} \overline{\mathbf{\Upsilon}}_{k}^{T}+2 \mathbf{A}_{o} \widetilde{\Xi}_{12 c, k}^{T} \overline{\mathbf{C}}_{k}^{T} \overline{\mathbf{\Upsilon}}_{k}^{T} \\
& -2 \mathbf{K}_{o}\left[\overline{\mathbf{\Upsilon}}_{k} \overline{\mathbf{C}}_{k}\left(\widetilde{\boldsymbol{\Xi}}_{12 c, k}+\widetilde{\boldsymbol{\Xi}}_{12 c, k}^{T}\right) \overline{\mathbf{C}}_{k}^{T} \overline{\mathbf{\Upsilon}}_{k}^{T}\right] \\
& -2\left(\mathbf{A}_{o}-\mathbf{K}_{o} \overline{\mathbf{\Upsilon}}_{k} \overline{\mathbf{C}}_{k}\right) \widetilde{\boldsymbol{\Xi}}_{22 c, k} \overline{\mathbf{C}}_{k}^{T} \overline{\mathbf{Y}}_{k}^{T} \\
& +2 \mathbf{K}_{o}\left[\overline{\mathbf{Y}}_{k}\left(\lambda_{1}^{-1} \overline{\mathbf{H}}_{2, k} \overline{\mathbf{H}}_{2, k}^{T}+\overline{\mathbf{R}}_{k}\right) \overline{\mathbf{\Upsilon}}_{k}^{T}\right. \\
& \left.+\boldsymbol{\Phi}_{1, k}+\boldsymbol{\Phi}_{2, k}\right]=0 .
\end{aligned}
$$

According to (59) and (60), the optimal parameters $\mathbf{A}_{o}$ and $\mathbf{K}_{o}$ to minimize the required upper bound can be calculated by

$$
\begin{gathered}
\mathbf{A}_{o}=\overline{\mathbf{A}}_{k}+\left(\overline{\mathbf{A}}_{k}-\mathbf{K}_{o} \overline{\mathbf{Y}}_{k} \overline{\mathbf{C}}_{k}\right)\left(\widetilde{\mathbf{\Xi}}_{12 c, k} \widetilde{\Xi}_{22 c, k}^{-1}-\mathbf{I}\right), \\
\mathbf{K}_{o}=\overline{\mathbf{A}}_{k} \mathbf{S}_{k} \overline{\mathbf{C}}_{k}^{T} \overline{\mathbf{Y}}_{k}^{T}\left[\overline{\mathbf{Y}}_{k} \overline{\mathbf{C}}_{k} \mathbf{S}_{k} \overline{\mathbf{C}}_{k}^{T} \overline{\mathbf{Y}}_{k}^{T}+\overline{\mathbf{Y}}_{k}\left(\lambda_{1}^{-1} \overline{\mathbf{H}}_{2, k} \overline{\mathbf{H}}_{2, k}^{T}+\overline{\mathbf{R}}_{k}\right)\right. \\
\left.\times \overline{\mathbf{Y}}_{k}^{T}+\boldsymbol{\Phi}_{1, k}+\boldsymbol{\Phi}_{2, k}\right]^{-1} .
\end{gathered}
$$

Then, substituting (61) and (62) into (55) and (56), we can obtain

$$
\begin{aligned}
\widetilde{\Xi}_{12, k+1}= & \widetilde{\Xi}_{12, k+1}^{T}=\widetilde{\Xi}_{22, k+1} \\
= & \overline{\mathbf{A}}_{k} \widetilde{\Xi}_{12 c, k} \widetilde{\Xi}_{22 c, k}^{-1} \widetilde{\Xi}_{12 c, k}^{T} \overline{\mathbf{A}}_{k}^{T}+\overline{\mathbf{A}}_{k} \mathbf{S}_{k} \overline{\mathbf{C}}_{k}^{T} \overline{\mathbf{\Upsilon}}_{k}^{T} \\
& \times\left[\overline{\mathbf{Y}}_{k} \overline{\mathbf{C}}_{k} \mathbf{S}_{k} \overline{\mathbf{C}}_{k}^{T} \overline{\mathbf{\Upsilon}}_{k}^{T}+\overline{\mathbf{\Upsilon}}_{k}\left(\lambda_{1}^{-1} \overline{\mathbf{H}}_{2, k} \overline{\mathbf{H}}_{2, k}^{T}+\overline{\mathbf{R}}_{k}\right) \overline{\mathbf{\Upsilon}}_{k}^{T}\right. \\
& \left.\quad+\boldsymbol{\Phi}_{1, k}+\boldsymbol{\Phi}_{2, k}\right]^{-1} \overline{\mathbf{Y}}_{k} \overline{\mathbf{C}}_{k} \mathbf{S}_{k}^{T} \overline{\mathbf{A}}_{k}^{T} .
\end{aligned}
$$

Thus, when $n=k+1,(47)$ is still valid. We can deduce that (50) is valid, for $n \in[0, N]$. From (47), we can know that $\bar{\Xi}_{k}=\widetilde{\Xi}_{11, k}-\widetilde{\Xi}_{22, k}$. Utilizing (57), we have

$$
\begin{aligned}
\widetilde{\Xi}_{12 c, k} \widetilde{\Xi}_{22 c, k}^{-1}-\mathbf{I}=\overline{\boldsymbol{\Xi}}_{k} \mathbf{G}_{k}^{T}\left(\lambda_{1}^{-1} \mathbf{I}-\mathbf{G}_{k} \overline{\mathbf{\Xi}}_{k} \mathbf{G}_{k}^{T}\right)^{-1} \mathbf{G}_{k}, \\
\mathbf{S}_{k}=\widetilde{\Xi}_{11 c, k}-\widetilde{\Xi}_{12 c, k} \widetilde{\boldsymbol{\Xi}}_{22 c, k}^{-1} \widetilde{\Xi}_{12 c, k}^{T} \\
=\overline{\boldsymbol{\Xi}}_{k}+\overline{\boldsymbol{\Xi}}_{k} \mathbf{G}_{k}^{T}\left(\lambda_{1}^{-1} \mathbf{I}-\mathbf{G}_{k} \overline{\boldsymbol{\Xi}}_{k} \mathbf{G}_{k}^{T}\right)^{-1} \mathbf{G}_{k} \overline{\boldsymbol{\Xi}}_{k} .
\end{aligned}
$$

Substituting (64) into (61), (48) can be given. Furthermore, using (59), the required upper bound $\bar{\Xi}_{k+1}$ can 
be rewritten as (56). Therefore, the theorem has been proved.

Based on the above theorems, the finite-horizon robust Kalman filter can be summarized as follows.

Step 1. The initial values can be given as $\widehat{\mathbf{X}}_{0}=\left[\begin{array}{ll}\mathbf{x}_{0}^{T} & 0\end{array}\right]^{T}$ and $\bar{\Xi}_{0}=\mathbf{U}_{0}$.

Step 2. In the presence of uncertainties and sensor delays, the parameters of the filter can be calculated by (48) and (49).

Step 3. According to (30) and (53), the state estimation $\widehat{\mathbf{X}}_{k}$ and the filtering error covariance $\bar{\Xi}_{k}$ can be obtained.

Remark 7. The finite-horizon robust Kalman filter design is accomplished by using Theorems 5 and 6 for uncertain attitude estimation system with star sensor delays. Different from the most existing attitude estimation filtering algorithms, the finite-horizon robust Kalman filter presented in this paper has the advantage to consider the misalignment errors and scale factor errors of gyros and measurement delays of star sensors for attitude estimation system. Note that these phenomena of the misalignment errors of gyro and star sensor and star sensor measurement delays are often encountered in real attitude estimation systems. To compensate the misalignment errors of sensor and star sensor measurement delays, we have designed a finite-horizon robust Kalman filter by finding the upper bound of the estimation error covariance and minimizing the upper bound. It is worth mentioning that, due to the presence of the star sensor measurement delays, the upper bound of the estimation error covariance in (35) and the filter parameters $\mathbf{A}_{o}$ and $\mathbf{K}_{o}$ in (48) and (49) distinguished our work from the counterpart in $[15,16]$. In addition, this paper talks about the filter problem with only one type of noise disturbance. The filter problem with multiple disturbances can be considered to achieve more practical oriented results, as discussed in $[28,29]$, which will be one of our future research topics.

\section{Simulations and Analysis}

4.1. Simulation Conditions. The simulation utilizes the data from a satellite. The initial orbit elements of the satellite are set as follows: the semimajor axis $a=7.087457 \times 10^{3} \mathrm{~km}$, the eccentricity $e=1.99 \times 10^{-3}$, the inclination $i=98.153^{\circ}$, the ascending node longitude $\Omega=-30.534^{\circ}$, and the argument of perigee $\omega^{*}=-0.133^{\circ}$. Numerical simulation gives the measurement data of gyro. The standard deviation of gyros' measurement noise is $\sigma_{v}=1.45444 \times 10^{-6} \mathrm{rad} / \mathrm{s}^{1 / 2}$; the standard deviation of gyros' drift noise is $\sigma_{u}=1.3036 \times$ $10^{-9} \mathrm{rad} / \mathrm{s}^{3 / 2}$; the gyro sampling interval is $\Delta t=0.25 \mathrm{~s}$; the components of the gyro scale factor error vector are chosen randomly at the interval $\left[-6 \times 10^{-6}, 6 \times 10^{-6}\right]$; the misalignment error of gyro is chosen randomly at the interval $\left[-3 \times 10^{-6}, 3 \times 10^{-6}\right]$; three star sensors are used and the standard deviation of star sensors' measurement noise is all $\sigma_{s}=18^{\prime \prime}$. The misalignment error of star sensor is set to $\left[-5^{\prime \prime}, 5^{\prime \prime}\right]$. Three star sensors have different delay rates, so the random variables $\mu_{k, i}(i=1,2, \ldots, 9)$ satisfy the Bernoulli distribution with

$$
\overline{\boldsymbol{\Gamma}}_{k}=\operatorname{diag}\{0.2,0.2,0.2,0.1,0.1,0.1,0.05,0.05,0.05\} .
$$

The initial attitude quaternion of the system is taken as $\mathbf{q}_{0}=\left[\begin{array}{llll}0 & 0 & 0 & 1\end{array}\right]^{T}$; the gyros' initial bias is set as $\boldsymbol{\beta}=$ $\left[\begin{array}{lll}0.1 & 0.1 & 0.1\end{array}\right]^{T^{\circ}} / h$; all the filters are initialized with no attitude errors and zero bias estimate; the initial attitude error covariance is set at $0.1^{\circ}$ for the quaternion components and $0.2^{\circ}$ for the bias components. In order to complete the robust filter design and ensure the estimation precision, the parameters $\lambda_{1}, \lambda_{2}$, and $\lambda_{3}$ are set to satisfy the condition (40).

4.2. Simulation Results. To validate the effectiveness of the proposed robust filter for controlling the measurement errors of gyros and star sensors and star sensor delays, the proposed approach (FRKF) is compared with the traditional Kalman filter (KF) and the robust Kalman filter (RKF) in the literature [15]. For a fair comparison, the root-mean square error (RMSE) and accumulative RMSE (ARMSE) [30, 31] of the attitude are employed to describe the quality of the attitude estimation. Monte-Carlo simulation runs are set as $N_{\mathrm{MC}}=$ 50 , and the RMSE of attitude angles can be defined by

$$
\operatorname{RMSE}_{\text {att }}(k)=\sqrt{\frac{1}{N_{\mathrm{MC}}} \sum_{i=1}^{N_{\mathrm{MC}}}\left\|\mathbf{a e}_{i}(k)\right\|^{2}},
$$

where $\mathbf{a e}_{i}(k)$ expresses the attitude estimation error vector at the $i$ th Monte-Carlo run. Then, the ARMSE of the attitude is defined by

$$
\mathrm{AMSE}_{\mathrm{att}}=\sqrt{\frac{1}{N} \sum_{i=1}^{N} \mathrm{RMSE}_{\mathrm{att}}^{2}(k),}
$$

where $N$ denotes the simulation time. The simulation results are shown in Figures 2-4.

From Figures 2 and 3, it is obvious to be seen that the FRKF performs much better than the RKF and KF, and the KF performs the worst. This is because the traditional KF is not suitable for handling the model uncertainties in the system model and star sensor delays. However, the RKF compensates the measurement errors of gyros and star sensors, whose precisions are better than the KF. But the RKF cannot control the effect of the star sensor delays. Compared with the RKF, the FRKF has higher estimation precision that the RKF, which indicates that the FRKF not only deals with the measurement errors of gyros and star sensors but also cope with the star sensor delays.

For the sake of accounting for the effect of star sensor delays, three star sensors are assumed to have the same delay rate, such as $p_{k, i}(i=1,2, \ldots, m)=p$. Figure 4 shows the ARMSE of the attitude angles from three filters when $p=$ $0,0.1,0.2, \ldots, 1.0$. From Figure 4 , it can be seen that if there are no star sensor delays, that is, $p=0$, the FRKF is equal to the RKF. Meanwhile, it is apparent that the KF and RKF 


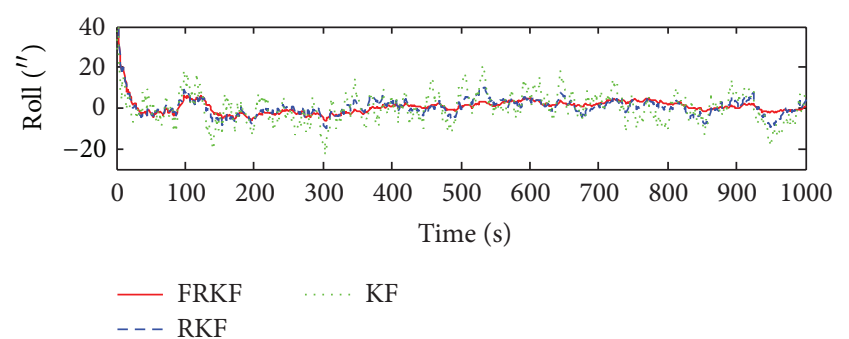

(a)

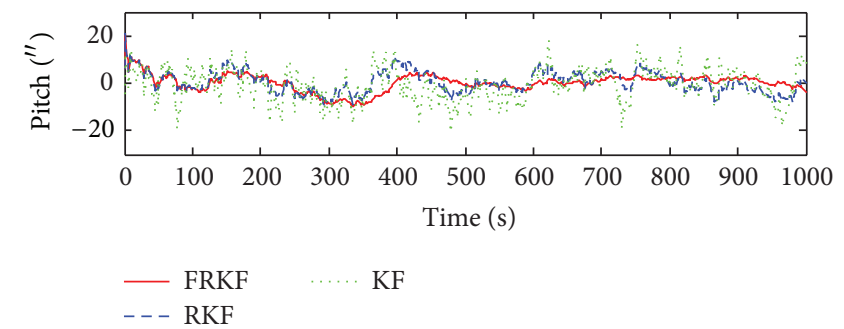

(b)

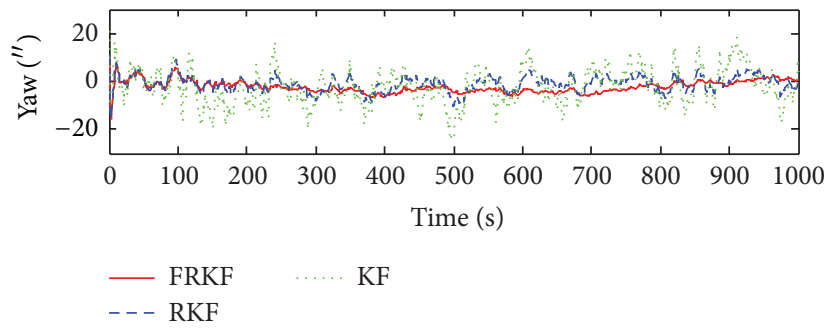

(c)

FIGURE 2: Attitude estimation errors.

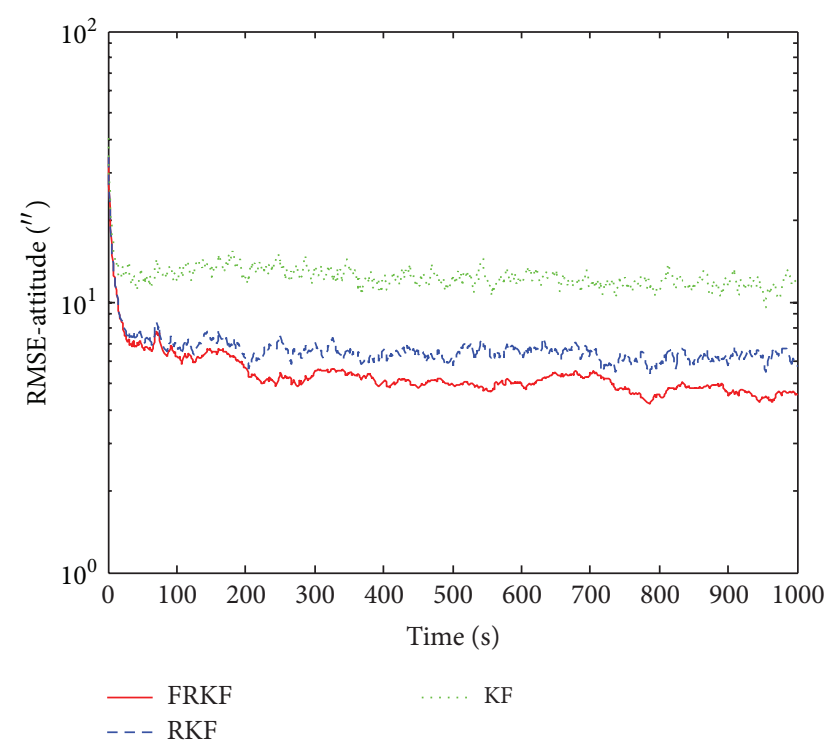

FIGURE 3: RMSE of the attitude angles.

increase faster than the FRKF as the delay probability $p$ is greater, which illustrates the efficiency of the FRKF to control the star sensor delays.

\section{Conclusion}

By the fact that the misalignment errors and scale factor errors of gyros and the misalignment errors of star sensors are difficult to be removed entirely by the attitude estimation filter calibration, these measurement errors of sensors are assumed as the norm-bounded uncertainties in the attitude estimation model. At the same time, due to the complicated signal processing of star sensors, the star sensor delay is

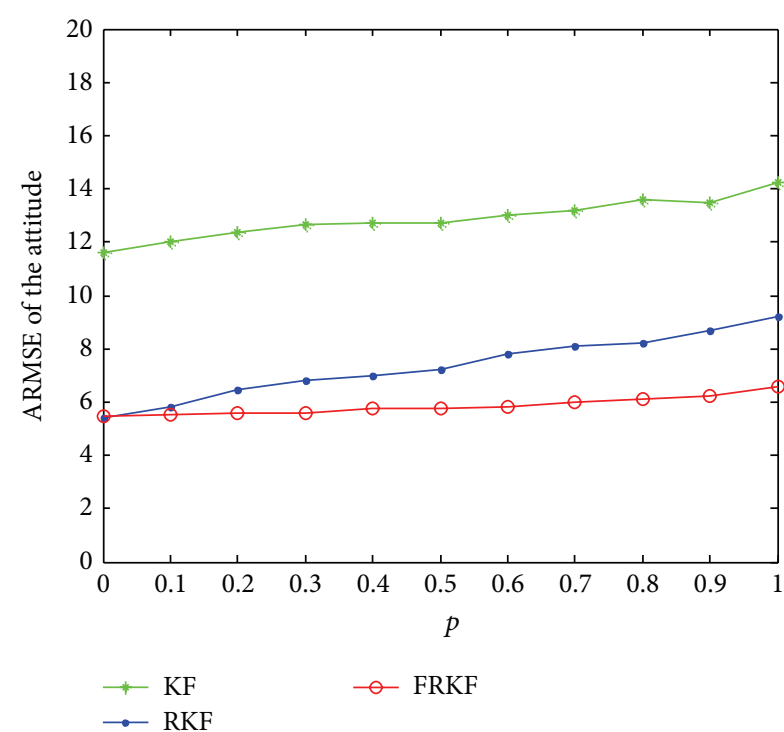

FIGURE 4: ARMSE of the attitude angles.

one of the most important problems for attitude estimation system. Therefore, a finite-horizon robust Kalman filter for the uncertain attitude estimation system with star sensor delays is proposed in this paper. The uncertain attitude estimation model with star sensor delays is constructed, and the finite-horizon robust Kalman filter design is developed. Finally, the applicability and effectiveness of the proposed filter have been demonstrated by the simulation.

\section{Conflict of Interests}

The authors declare that there is no conflict of interests regarding the publication of this paper. 


\section{References}

[1] E. J. Lefferts, F. L. Markley, and M. D. Shuster, "Kalman filtering for spacecraft attitude estimation," Journal of Guidance, Control, and Dynamics, vol. 5, no. 5, pp. 417-429, 1982.

[2] F. L. Markley, "Attitude error representations for Kalman filtering," Journal of Guidance, Control, and Dynamics, vol. 26, no. 2, pp. 311-317, 2003.

[3] J. L. Crassidis, F. L. Markley, and Y. Cheng, "Survey of nonlinear attitude estimation methods," Journal of Guidance, Control, and Dynamics, vol. 30, no. 1, pp. 12-28, 2007.

[4] M. D. Shuster, D. S. Pitone, and G. J. Bierman, "Batch estimation of spacecraft sensor alignments-relative alignment estimation," Journal of the Astronautical Sciences, vol. 39, no. 4, pp. 519546, 1991.

[5] M. Pittelkau, "Kalman filtering for spacecraft system alignment calibration," Journal of Guidance, Control, and Dynamics, vol. 24, no. 6, pp. 1187-1195, 2001.

[6] M. Pittelkau, "Survey of calibration algorithms for spacecraft attitude sensors and gyros," Advances in the Astronautical Sciences, vol. 129, paper no. AAS 07-295, pp. 651-706, 2008.

[7] K. L. Lai and J. L. Crassidis, "In-space spacecraft alignment calibration using the unscented filter," in Proceedings of the AIAA Guidance, Navigation, and Control Conference and Exhibit, pp. 1-11, 2003.

[8] J. Vandersteen, M. Diehl, C. Aerts, and J. Swevers, "Spacecraft attitude estimation and sensor calibration using moving horizon estimation," Journal of Guidance, Control, and Dynamics, vol. 36, no. 3, pp. 734-742, 2013.

[9] A. Hmamed, C. E. Kasri, E. H. Tissir, T. Alvarez, and F. Tadeo, "Robust $H_{\infty}$ filtering for uncertain 2-D continuous systems with delays," International Journal of Innovative Computing, Information and Control, vol. 9, no. 5, pp. 2167-2183, 2013.

[10] C. K. Ahn and P. S. Kim, "New energy-to-peak FIR filter design for systems with disturbance: a convex optimization approach," International Journal of Innovative Computing, Information and Control, vol. 9, no. 5, pp. 1987-1993, 2013.

[11] L. G. Wu and D. W. C. Ho, "Fuzzy filter design for Itô stochastic systems with application to sensor fault detection," IEEE Transactions on Fuzzy Systems, vol. 17, no. 1, pp. 233-242, 2009.

[12] J. Hu, Z. D. Wang, H. J. Gao, and L. K. Stergioulas, "Extended Kalman filtering with stochastic nonlinearities and multiple missing measurements," Automatica, vol. 48, no. 9, pp. 20072015, 2012.

[13] J. Hu, Z. D. Wang, B. Shen, and H. J. Gao, "Quantised recursive filtering for a class of nonlinear systems with multiplicative noises and missing measurements," International Journal of Control, vol. 86, no. 4, pp. 650-663, 2013.

[14] J. Q. Wang, Z. M. He, H. Y. Zhou, and Y. Y. Jiao, "Regularized robust filter for attitude determination system with relative installation error of star trackers," Acta Astronautica, vol. 87, pp. 88-95, 2013.

[15] Z. Dong and Z. You, "Finite-horizon robust Kalman filtering for uncertain discrete time-varying systems with uncertaincovariance white noises," IEEE Signal Processing Letters, vol. 13, no. 8, pp. 493-496, 2006.

[16] R. F. Souto and J. Y. Ishihara, "Comments on 'finite: horizon robust Kalman filtering for uncertain discrete time: varying systems with uncertain: covariance white noises"' IEEE Signal Processing Letters, vol. 17, no. 2, pp. 213-216, 2010.
[17] X. Su, L. Wu, and P. Shi, "Sensor networks with random link failures: distributed filtering for T-S fuzzy systems," IEEE Transactions on Industrial Informatics, vol. 9, no. 3, pp. 17391750, 2013.

[18] X. Su, P. Shi, L. Wu, and S. K. Nguang, "Induced $l_{2}$ filtering of fuzzy stochastic systems with time-varying delays," IEEE Transactions on Cybernetics, vol. 43, no. 4, pp. 1251-1264, 2013.

[19] R. Yang, P. Shi, and G. Liu, "Filtering for discrete-time networked nonlinear systems with mixed random delays and packet dropouts," IEEE Transactions on Automatic Control, vol. 56, no. 11, pp. 2655-2660, 2011.

[20] R. Caballero-Aguila, A. Hermoso-Carazo, J. D. Jimenez-Lopez, J. Linares-Perez, and S. Nakamori, "Recursive estimation of discrete-time signals from nonlinear randomly delayed observations," Computers \& Mathematics with Applications, vol. 58, no. 6, pp. 1160-1168, 2009.

[21] R. Caballero-Aguilaa, A. Hermoso-Carazo, J. D. Jimenez-Lopez, J. Linares-Perez, and S. Nakamori, "Signal estimation with multiple delayed sensors using covariance information," Digital Signal Processing, vol. 20, no. 2, pp. 528-540, 2010.

[22] H. H. Dam, "Variable fractional delay filter with sub-expression coefficients," International Journal of Innovative Computing, Information and Control, vol. 9, no. 7, pp. 2995-3003, 2013.

[23] F. O. Hounkpevi and E. Yaz, "Minimum variance generalized state estimators for multiple sensors with different delay rates," Signal Processing, vol. 87, no. 4, pp. 602-613, 2007.

[24] X. X. Wang, Y. Liang, Q. Pan, and C. H. Zhao, "Gaussian filter for nonlinear systems with one-step randomly delayed measurements," Automatica, vol. 49, no. 4, pp. 976-986, 2013.

[25] J. Hu, Z. D. Wang, B. Shen, and H. J. Gao, "Gain-constrained recursive filtering with stochastic nonlinearities and probabilistic sensor delays," IEEE Transactions on Signal Processing, vol. 61, no. 5, pp. 1230-1238, 2013.

[26] L. Xie, Y. C. Soh, and C. E. de Souza, "Robust Kalman filtering for uncertain discrete-time systems," IEEE Transactions on Automatic Control, vol. 39, no. 6, pp. 1310-1314, 1994.

[27] R. A. Horn and C. R. Johnson, Topics in Matrix Analysis, Cambridge University Press, New York, NY, USA, 1991.

[28] X. M. Yao and L. Guo, "Composite anti-disturbance control for Markovian jump nonlinear systems via disturbance observer," Automatica, vol. 49, no. 8, pp. 2538-2545, 2013.

[29] X. M. Yao, Z. Dong, and D. F. Wang, "Full-order disturbanceobserver-based control for singular hybrid system," Mathematical Problems in Engineering, Article ID 352198, 7 pages, 2013.

[30] X. J. Tang, Z. B. Liu, and J. S. Zhang, "Square-root quaternion cubature Kalman filtering for spacecraft attitude estimation," Acta Astronautica, vol. 76, pp. 84-94, 2012.

[31] I. Arasaratnam, S. Haykin, and T. R. Hurd, "Cubature Kalman filtering for continuous-discrete systems: theory and simulations," IEEE Transactions on Signal Processing, vol. 58, no. 10, pp. 4977-4993, 2010. 


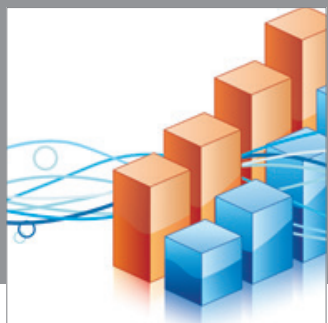

Advances in

Operations Research

mansans

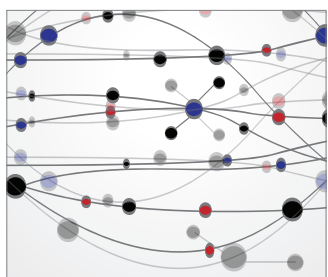

The Scientific World Journal
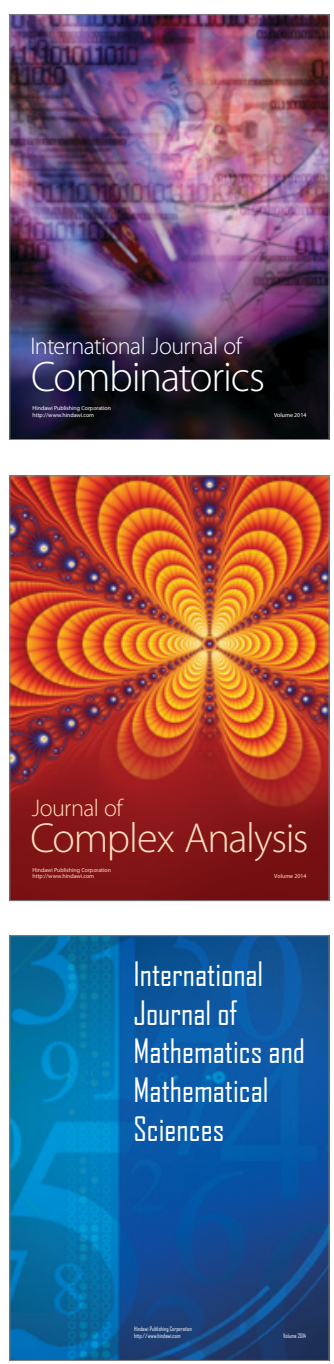
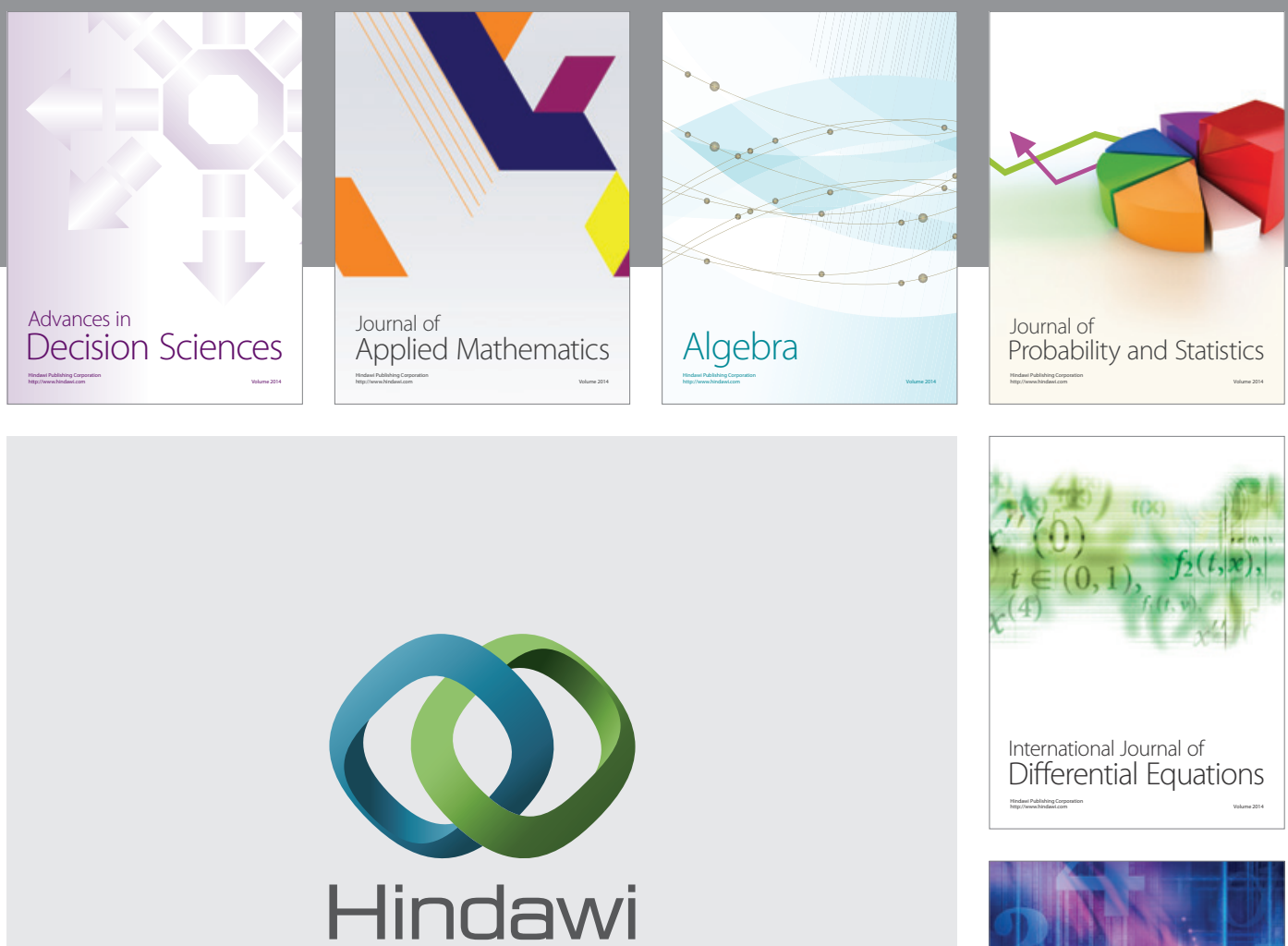

Submit your manuscripts at http://www.hindawi.com
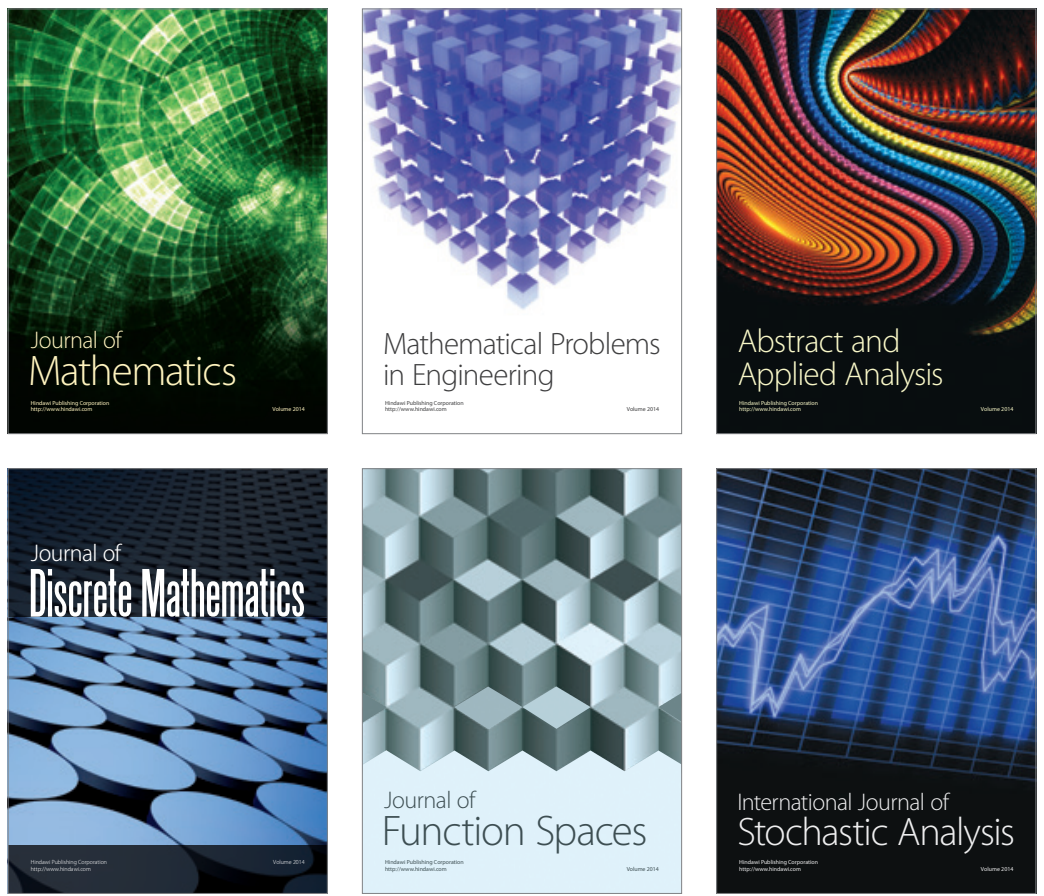

Journal of

Function Spaces

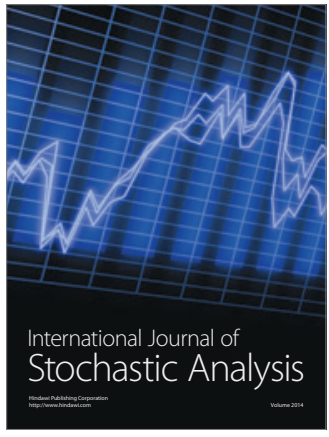

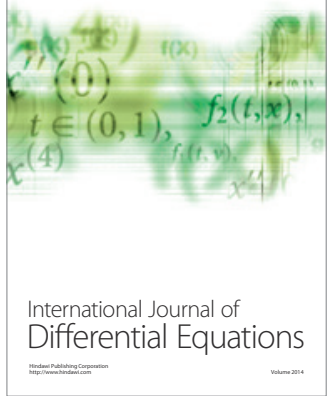
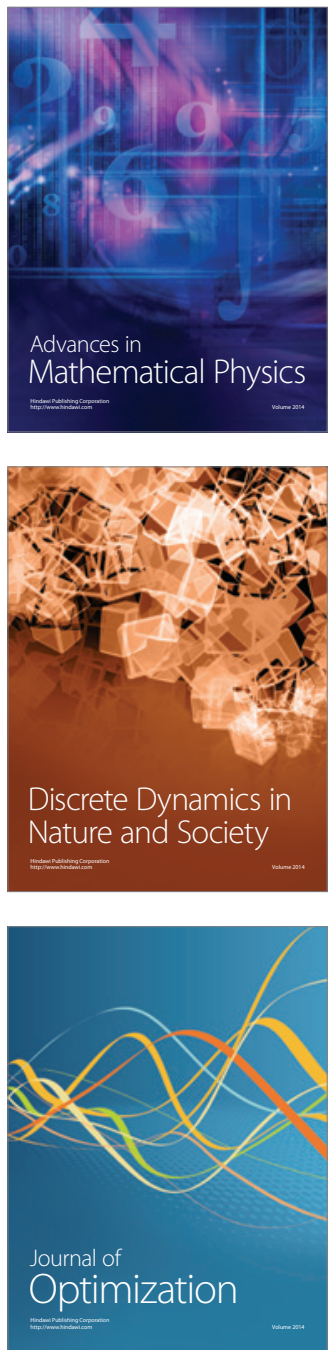